Document downloaded from:

http://hdl.handle.net/10251/83360

This paper must be cited as:

Blasco Ferragud, FX.; Reynoso Meza, G.; Sánchez Pérez, EA.; Sánchez Pérez, JV. (2016). Asymmetric distances to improve $\mathrm{n}$-dimensional Pareto fronts graphical analysis. Information Sciences. 340:228-249. doi:10.1016/j.ins.2015.12.039.

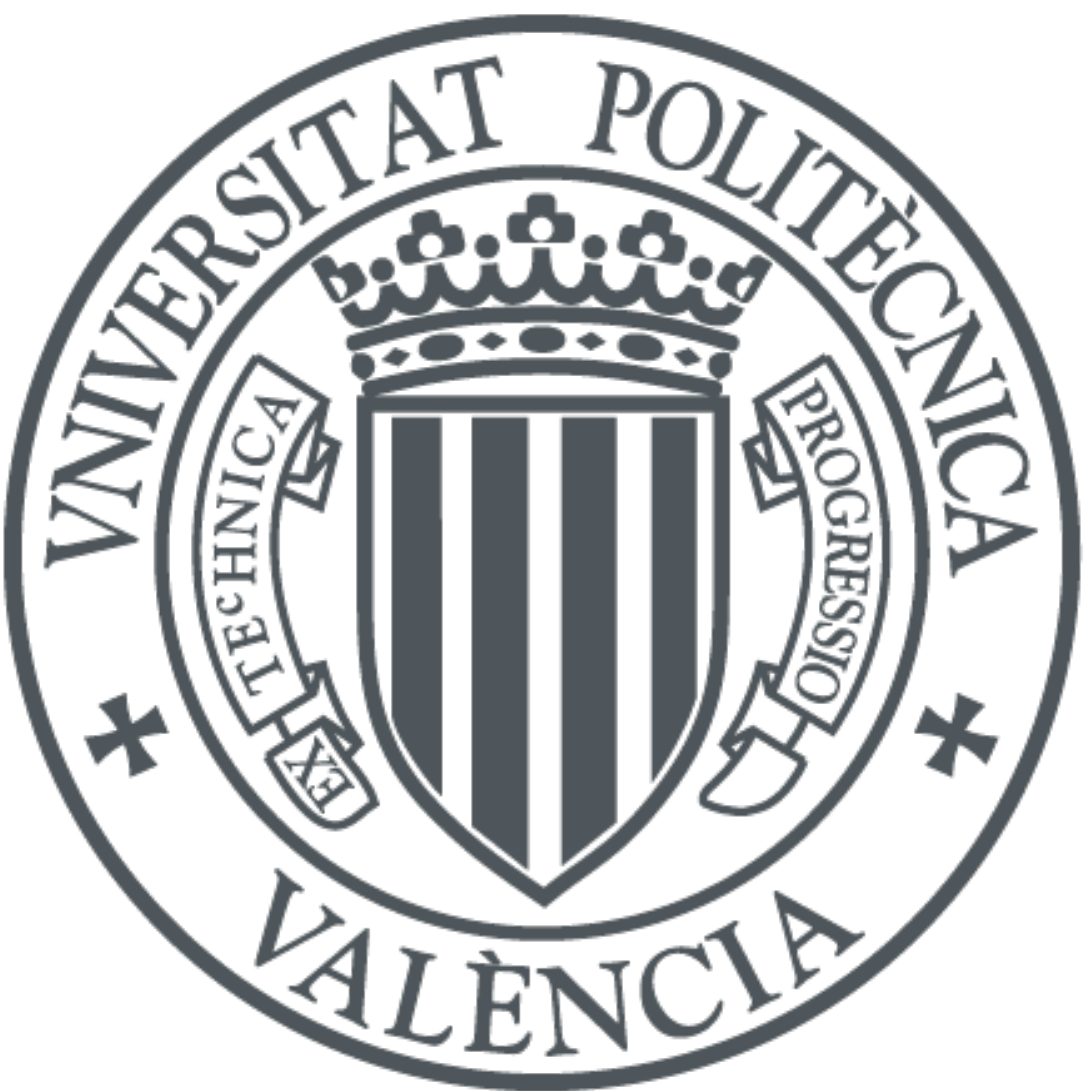

The final publication is available at

http://doi.org/10.1016/j.ins.2015.12.039

Copyright Elsevier

Additional Information 


\title{
Asymmetric distances to improve $n$-dimensional Pareto fronts graphical analysis
}

\author{
Xavier Blasco ${ }^{a, *}$, Gilberto Reynoso-Meza ${ }^{\text {b }}$, Enrique A. Sánchez Pérez ${ }^{\mathrm{c}}$, Juan V. Sánchez \\ Pérez ${ }^{\mathrm{d}}$ \\ ${ }^{a}$ Instituto Universitario de Automática e Informática Industrial, Universitat Politècnica de València, \\ Camino de Vera $S / N$, Valencia 46022, Spain \\ ${ }^{b}$ Programa de Pós-Graduação em Engenharia de Produção e Sistemas (PPGEPS), Pontificia Universidade \\ Católica do Paraná (PUCPR), Imaculada Conceição 1155, Curitiba 80215-901, Brazil \\ ${ }^{c}$ Instituto Universitario de Matemática Pura y Aplicada (IUMPA), Universitat Politècnica de València, \\ Camino de Vera $S / N$, Valencia 46022, Spain \\ ${ }^{d}$ Centro de Tecnologías Físicas: Acústica, Materiales y Astrofísica (CTF:AMA), Universitat Politècnica de \\ València, Camino de Vera S/N, Valencia 46022, Spain
}

\begin{abstract}
Visualization tools and techniques to analyze $n$-dimensional Pareto fronts are valuable for designers and decision makers in order to analyze straightness and drawbacks among design alternatives. Their usefulness is twofold: on the one hand, they provide a practical framework to the decision maker in order to select the preferable solution to be implemented; on the other hand, they may improve the decision maker's design insight, i.e. increasing the designer's knowledge on the multi-objective problem at hand. In this work, an order based asymmetric topology for finite dimensional spaces is introduced. This asymmetric topology, associated to what we called asymmetric distance, provides a theoretical and interpretable framework to analyze design alternatives for $n$-dimensional Pareto fronts. The use of this asymmetric distance will allow a new way to gather dominance and relative distance together. This property can be exploited inside interactive visualization tools. Additionally, a composed norm based on asymmetric distance has been developed. The composed norm allows a fast visualization of designer preferences hypercubes when Level Diagram visualization is used for multidimensional Pareto front analysis. All these proposals are evaluated and validated through different engineering benchmarks; the presented results show the usefulness of this asymmetric topology to improve visualization interpretability.
\end{abstract}

Keywords: multi-criteria decision-making, asymmetric distance, multi-objective optimization, decision-making tools, $n$-dimensional Pareto front, $n$-dimensional Pareto front visualization.

\footnotetext{
${ }^{*}$ Corresponding author

Email addresses: xblasco@isa.upv.es (Xavier Blasco ), g.reynosomeza@pucpr.br (Gilberto Reynoso-Meza ), easancpe@mat.upv.es (Enrique A. Sánchez Pérez ), jusanc@fis.upv.es (Juan V. Sánchez Pérez )
} 


\section{Introduction}

It is common to state a design problem as an optimization statement, where a specific cost index must be optimized. However, many real world problems require the fulfillment of a set of requirements and specifications. In that case, it is said to have a multi-objective problem (MOP) instead of a single-objective problem. In such statements, it is usual to find that some objectives are in conflict with each other, and therefore a trade-off solution must be found (or selected).

Multi-objective optimization (MOO) can handle these issues in a simple manner, due to its simultaneous optimization approach. In MOO, all the objectives are significant to the designer, and as a consequence, each is optimized. In general, there is no a single solution because no solution is better than the others in all the objectives. Therefore, a set of solutions, the Pareto set $\boldsymbol{\Theta}_{\boldsymbol{P}}$, is defined and its objective vector set is the Pareto front $\boldsymbol{J}_{\boldsymbol{P}}$. This set of solutions offers to the decision maker (DM) greater flexibility at the multi-criteria decision-making (MCDM) stage. The role of the designer is to select the best solution according to her/his needs and preferences for a particular situation.

MOO techniques search for a discrete approximation $\boldsymbol{\Theta}_{\boldsymbol{P}}^{*}$ of the Pareto set $\boldsymbol{\Theta}_{\boldsymbol{P}}$ in order to build a useful description $\boldsymbol{J}_{\boldsymbol{P}}^{*}$ of the Pareto front that is as good as possible, according to the DM needs. In this way, the DM has a set of solutions for a given problem and a high degree of flexibility when choosing a particular or desired solution. Classic techniques [26] for ding this Pareto front have been proposed and multi-objective evolutionary algorithms (MOEAs) have been recently used due to their flexibility when dealing with non-convex and highly constrained functions [9, 8].

Once the DM has been provided with a Pareto front approximation $\boldsymbol{J}_{P}^{*}$, the DM will need to analyze the trade-off between objectives with two aims: firstly, in order to select the most preferable solution according to her/his preferences: secondly, in order to gain a better design insight of the MOP by innovization, that is innovation trough optimization [11.

Several techniques and methods have been developed to facilitate the DM's task [12, 2, 27, 36]. It is widely accepted that visualization tools are valuable and provide to the DM meaningful methods to analyze the Pareto front and take decisions [4, 34]. We can recall the desirable characteristics for such visualization techniques noted in [21]: simplicity (it should be easy to understand); persistence (the DM should be able to retain all information in his/her mind); and completeness (all the relevant information should be shown). Moreover, desirable characteristics (at software level) include interactivity with the DM and an intuitive graphical user interface.

For two-dimensional problems (and sometimes for three-dimensional problems) it is usually straightforward to make an accurate graphical analysis of the Pareto front, but the difficulty increases with the dimension of the problem. Common alternatives to tackle an analysis in higher dimensions are Scatter Diagrams, Parallel Coordinates [17, 18] and Level Diagrams (LD) [3, 30]. Recently, hybrid tools merging Parallel Coordinates, Dendrograms, and Cluster Maps have been proposed [5]. Nevertheless, in spite of the usefulness of those tools in their own merits, new approaches and efforts to improve interpretability and allowing the DM to perform an accurate analysis are valuable. 
Recently, asymmetric distances have become a useful and efficient tool for theoreticians and engineers; their intrinsic properties, allowing to state that the distance from $A$ to $B$ is not the same as the one from $B$ to $A$, make them a flexible tool to handle problems in a wide variety of domains. They have been successfully used for computer science applications [24], embedding techniques [16], clustering [28, 6] and visualizing asymmetric proximity in self organizing maps and multi-dimensional scaling [22]. Their success is mainly due to their capabilities to incorporate asymmetric relations between data, quite common characteristic in real world applications problems. Therefore, incorporating asymmetric distances into visualization techniques, in order to facilitate the DM's analysis of $n$-dimensional Pareto fronts, could bring an interesting insight, closer to the point of view of the designer improving persistency and completeness.

In this paper, a new order based asymmetric topology is introduced to carry out an analysis between design alternatives for a given MOP. The use of this asymmetric distance (associated to the asymmetric topology) gives a new way to gather dominance and relative distance together with a lower computational cost than the traditional way. This property can be exploited inside interactive visualization tools. Used for coloring purpose it is possible to apply it to several type of visualization, for instance, parallel coordinates, star diagrams, level diagrams, etc. Each visualization technique has its own particular properties. In this article Level Diagrams is used to demonstrate the application of coloring methods based on asymmetric distance. Additionally, a composed norm based on asymmetric distance has been developed. The composed norm allows a fast visualization of designer preferences hypercubes when Level Diagram visualization is used for multidimensional Pareto front analysis. This work is developed on the following assumptions:

- The DM has chosen to tackle a MOP by means of MOO in order to approximate a Pareto front; therefore, this optimization process will provide a set of Pareto optimal solutions, in order to perform a MCDM stage. That is, it is difficult to find the desirable trade-off with other techniques.

- For such MCDM stage, the DM is willing to analyze trade-off among design alternatives, in order to select the most preferable solution according to her/his needs. That is, any (semi)automatic selection procedure will be used.

The remainder of this paper is as follows: in Section 2 some preliminaries and background are stated. In Section 3 the new order based asymmetric topology is defined for $n$-dimensional Pareto front visualization and the coloring procedures are described. The composed norm for Level Diagram synchronization is presented. In Section 4, this topology is used in several instances, in order to validate its usability for such analysis. Finally, some concluding remarks are given. 


\section{Background}

\subsection{Pareto Optimality}

A MOP, without loss of generality $\left.\right|^{1}$ can be stated as follows:

$$
\begin{array}{ll} 
& \min _{\boldsymbol{\theta}} \boldsymbol{J}(\boldsymbol{\theta}) \\
\text { subject to: } & \boldsymbol{\theta} \in S \subset \mathbb{R}^{m}
\end{array}
$$

where $\boldsymbol{\theta}=\left(\theta_{1}, \ldots, \theta_{m}\right) \in \mathbb{R}^{m}$ is defined as the decision vector, $\boldsymbol{J}(\boldsymbol{\theta})=\left(J_{1}(\boldsymbol{\theta}), \ldots, J_{n}(\boldsymbol{\theta})\right) \in$ $\mathbb{R}^{n}$ as the objective vector, and $S$ as the subspace that satisfies all the additional constraints of the problem.

In this type of problem some of the objectives are in conflict, and therefore there is no a single better solution because no solution is the best in all objectives. Pareto optimality is the base to define an optimal set.

It is said that a decision vector $\boldsymbol{\theta}^{1}$ dominates (Pareto dominance) another vector $\boldsymbol{\theta}^{2}$, denoted as $\boldsymbol{\theta}^{1} \prec \boldsymbol{\theta}^{2}$, if $\boldsymbol{J}\left(\boldsymbol{\theta}^{1}\right)$ is not worse than $\boldsymbol{J}\left(\boldsymbol{\theta}^{2}\right)$ in all objectives and is better in at least one objective.

$$
\boldsymbol{\theta}^{1} \prec \boldsymbol{\theta}^{2} \rightarrow \forall i \in A:=[1, \ldots, n], J_{i}\left(\boldsymbol{\theta}^{\mathbf{1}}\right) \leq J_{i}\left(\boldsymbol{\theta}^{\mathbf{2}}\right) \wedge \exists i \in A: J_{i}\left(\boldsymbol{\theta}^{\mathbf{1}}\right)<J_{i}\left(\boldsymbol{\theta}^{\mathbf{2}}\right)
$$

A decision vector $\boldsymbol{\theta}^{*}$ is Pareto optimal if no other vector dominates it:

$$
\boldsymbol{\theta}^{*} \text { is Pareto optimal } \Longleftrightarrow \nexists \boldsymbol{\theta} \in S: \boldsymbol{\theta} \prec \boldsymbol{\theta}^{*}
$$

Therefore the set of optimal solutions, the Pareto set $\boldsymbol{\Theta}_{P}$ (set of all the non-dominated solutions) and its corresponding Pareto front $\boldsymbol{J}_{P}$, are defined as follow:

$$
\begin{gathered}
\boldsymbol{\Theta}_{P}:=\left\{\boldsymbol{\theta} \in S \mid \nexists \boldsymbol{\theta}^{\prime} \in S: \boldsymbol{\theta}^{\prime} \prec \boldsymbol{\theta}\right\} \\
\boldsymbol{J}_{P}:=\left\{\boldsymbol{J}(\boldsymbol{\theta}): \boldsymbol{\theta} \in \boldsymbol{\Theta}_{P}\right\}
\end{gathered}
$$

Figure 1 shows the principal concepts and definitions involved in a MOP. To simplify the graphical representation a bi-dimensional problem is shown for both decision and objective spaces. In the following, in order to simplify the notation, a particular point in the objective space will be denoted as $\boldsymbol{J}\left(\boldsymbol{\theta}^{1}\right)=J^{1}$ and consequently the coordinates of this point as $\left(J_{1}\left(\boldsymbol{\theta}^{1}\right), \ldots, J_{n}\left(\boldsymbol{\theta}^{1}\right)=\left(J_{1}^{1}, \ldots, J_{n}^{1}\right)\right)$.

\subsection{Asymmetric distance on the space $\mathbb{R}^{n}$.}

Asymmetric distances constitute a new mathematical topic that lies among the general topology and the functional analysis. It deals with the analysis of non symmetric topologies on linear spaces. Nowadays the fundamental results of the theory are already known, and

\footnotetext{
${ }^{1} \mathrm{~A}$ maximization problem can be converted to a minimization problem. For each of the objectives that has to be maximized, the transformation: $\max J_{i}(\boldsymbol{\theta})=-\min \left(-J_{i}(\boldsymbol{\theta})\right)$ could be applied.
} 

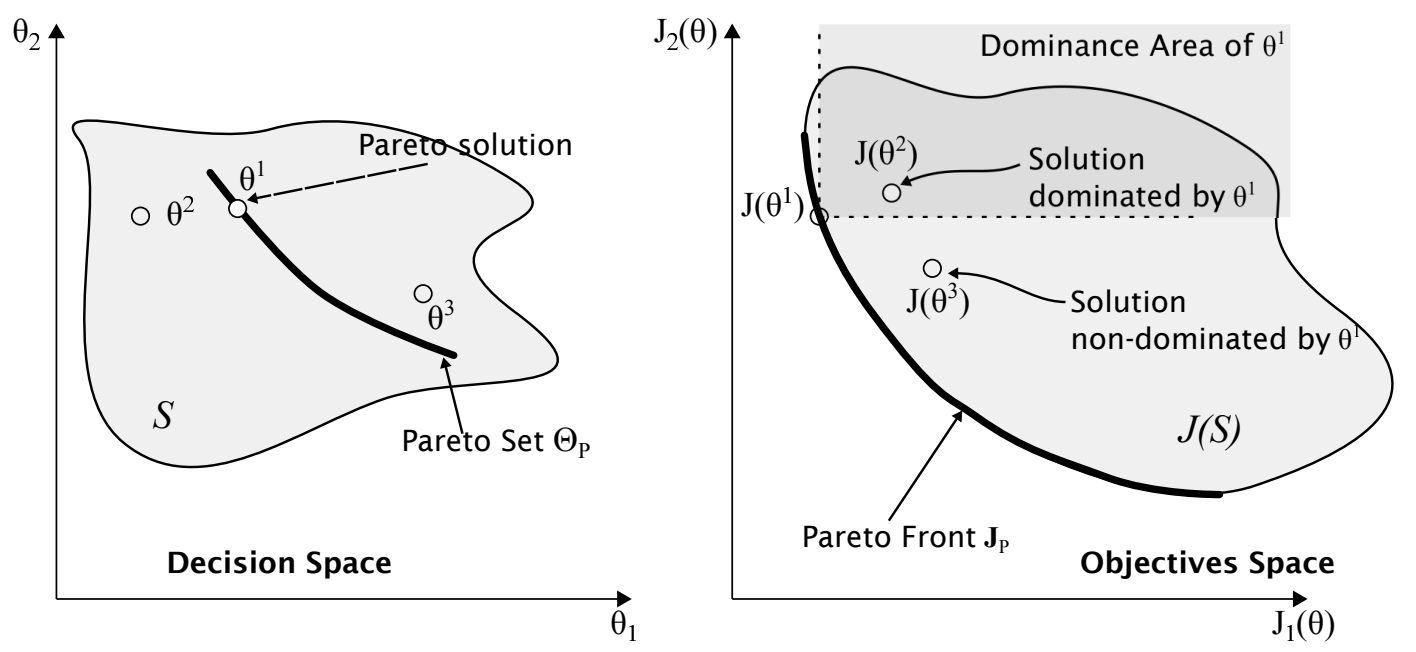

Figure 1: Representation of Multiobjective problem concepts (Dominance, Pareto Front and Pareto Set) for $2 \mathrm{D}$ decision and objective spaces.

it has been used with success in several areas, mainly in theoretical computer science. The interested reader can find a short explanation of this topic and some useful references in the Appendix at the end of the paper.

Let us define the particular asymmetric distance on $\mathbb{R}^{n}$ that will be used in this paper. Roughly speaking, an asymmetric distance is a positive function that allows to measure distances among points of $\mathbb{R}^{n}$ but for which the distance $d\left(J^{1}, J^{2}\right)$ from a given point $J^{1}$ to other point $J^{2}$ does not coincide necessarily with the distance $d\left(J^{2}, J^{1}\right)$ from $J^{2}$ to $J^{1}$. As in the case of Euclidean distance in $\mathbb{R}^{n}$, an asymmetric distance can be defined on a vector space by means of an asymmetric norm, as we do in this paper. Consider the coordinate-wise order $\leq$ on $\mathbb{R}^{n}$ and consider the Euclidean norm $\|\cdot\|$ on it. We write $\left(\mathbb{R}^{n}\right)^{+}$for the positive cone, that is, the set of all the elements of $\mathbb{R}^{n}$ that are coordinate-wise bigger or equal than 0 . Write $r^{+}=\max \{r, 0\}$ for $r \in \mathbb{R}$. If $J^{1}=\left(J_{1}^{1}, \ldots, J_{n}^{1}\right), J^{2}=\left(J_{1}^{2}, \ldots, J_{n}^{2}\right) \in \mathbb{R}^{n}$, we define the asymmetric Euclidean distance as

$$
d\left(J^{1}, J^{2}\right):=\left\|\left(J^{2}-J^{1}\right) \vee 0\right\|=\sqrt{\sum_{i=1}^{n}\left(\left(J_{i}^{2}-J_{i}^{1}\right)^{+}\right)^{2}}
$$

The same definition makes sense if we consider a different lattice norm on it instead of Euclidean norm, for example the 1-norm or the $\infty$-norm.

The sets $\Theta_{0}(J)$ of distance 0 of a given element $J \in \mathbb{R}^{n}$ will be often used in this paper. They are defined as

$$
\Theta_{0}\left(J^{0}\right):=\left\{J \in X: d\left(J^{0}, J\right)=0\right\} .
$$

It is easy to see that

$$
\Theta_{0}\left(J^{0}\right)=\left\{J \in \mathbb{R}^{n}: J \leq J^{0}\right\}
$$


That is, the set $\Theta_{0}(y)$ of asymmetric distance 0 to a given point $y$ of the space is defined exactly by the elements that dominate $y$. This geometric interpretation will be crucial for the optimization arguments of this paper. If we are considering a pair of points or solutions of a given optimization problem, the fact that $\boldsymbol{\theta}^{1}$ dominates $\boldsymbol{\theta}^{2}\left(\boldsymbol{\theta}^{1} \prec \boldsymbol{\theta}^{2}\right)$ - that is, that $\boldsymbol{\theta}^{1}$ is a better solution to the problem than $\boldsymbol{\theta}^{2}$-, can be written using our new tool as follows:

- $J\left(\boldsymbol{\theta}^{1}\right) \in \Theta_{0}\left(J\left(\boldsymbol{\theta}^{2}\right)\right)$ or $J^{1} \in \Theta_{0}\left(J^{2}\right)$.

- $d\left(J\left(\boldsymbol{\theta}^{2}\right), J\left(\boldsymbol{\theta}^{1}\right)\right)=0$ or $d\left(J^{2}, J^{1}\right)=0$.

Moreover, as we will explain later, the numerical value of $d\left(J^{2}, J^{1}\right)$ provides a metric notion of how far is $J^{1}$ of dominating $J^{2}$. Note also that for every $J \in \mathbb{R}^{n}, \Theta_{0}(J)=J+\Theta_{0}$, where $\Theta_{0}:=\Theta_{0}(0)$. That is, the set of distance 0 of a point $J$ is the sum of the element $J$ plus the "negative cone" of the lattice $\mathbb{R}^{n}$.

\subsection{Level Diagrams}

The Level Diagram (LD) visualization [3, 30] $]^{2}$ is useful for analyzing $n$-objective Pareto fronts and their corresponding Pareto sets. Each objective $J_{i}(\boldsymbol{\theta})(i \in[1, \ldots, n])$ is normalized $\hat{J}_{i}(\boldsymbol{\theta})$ with respect to some minimum and maximum values (by default the values of the Pareto front approximation are used). For each normalized objective vector $\hat{\boldsymbol{J}}(\boldsymbol{\theta})$ a norm $\left(\|\cdot\|_{p}\right)$ is applied to evaluate the distance to an ideal solution $\boldsymbol{J}^{\text {ideal }}$. By default, the minimal values for each objective in the Pareto front approximation could be used to build an ideal solution. 140 Then, the normalized value of the ideal point corresponds to the origin, $\hat{\boldsymbol{J}}^{\text {ideal }}=(0, \ldots, 0)$, and consequently, $\|\hat{\boldsymbol{J}}(\boldsymbol{\theta})\|_{p}=\left\|\hat{\boldsymbol{J}}(\boldsymbol{\theta})-\hat{\boldsymbol{J}}^{\text {ideal }}(\boldsymbol{\theta})\right\|_{p}$.
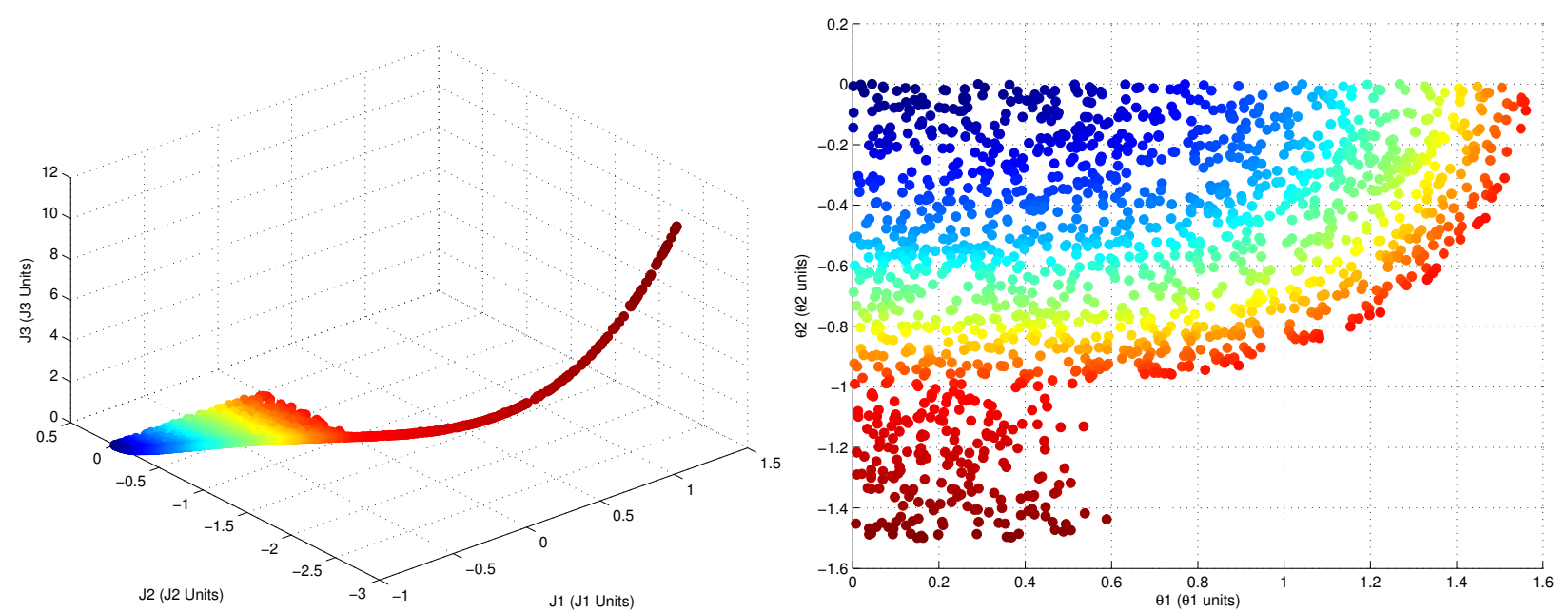

Figure 2: 3D visualization for a Pareto front and 2D visualization for Pareto set. Three objectives $([J 1, J 2, J 3])$ and two decision parameters $([\theta 1, \theta 2])$.

${ }^{2}$ GUI for Matlab(c) is available at: http://www.mathworks.com/matlabcentral/fileexchange/24042 

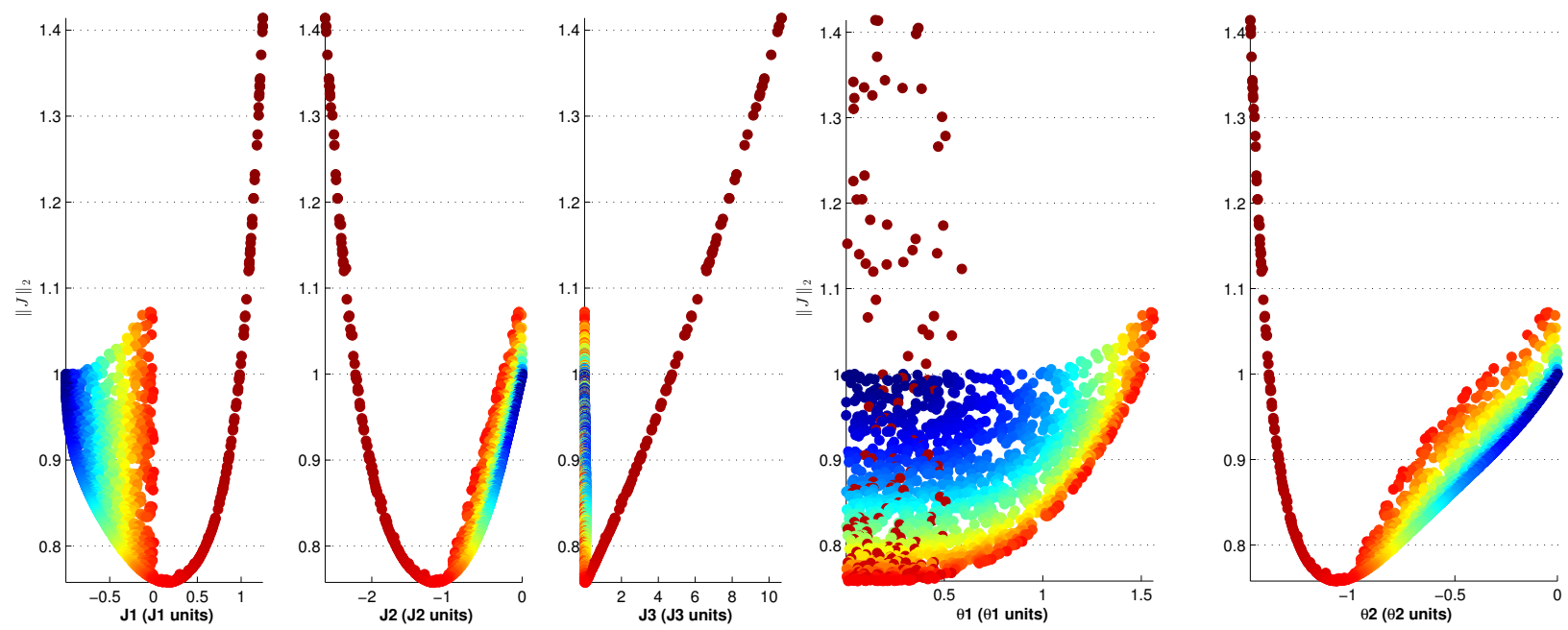

Figure 3: Level Diagram (LD) visualization for a Pareto front. Three objectives ([J1,J2,J3]) and two decision parameters $([\theta 1, \theta 2])$.
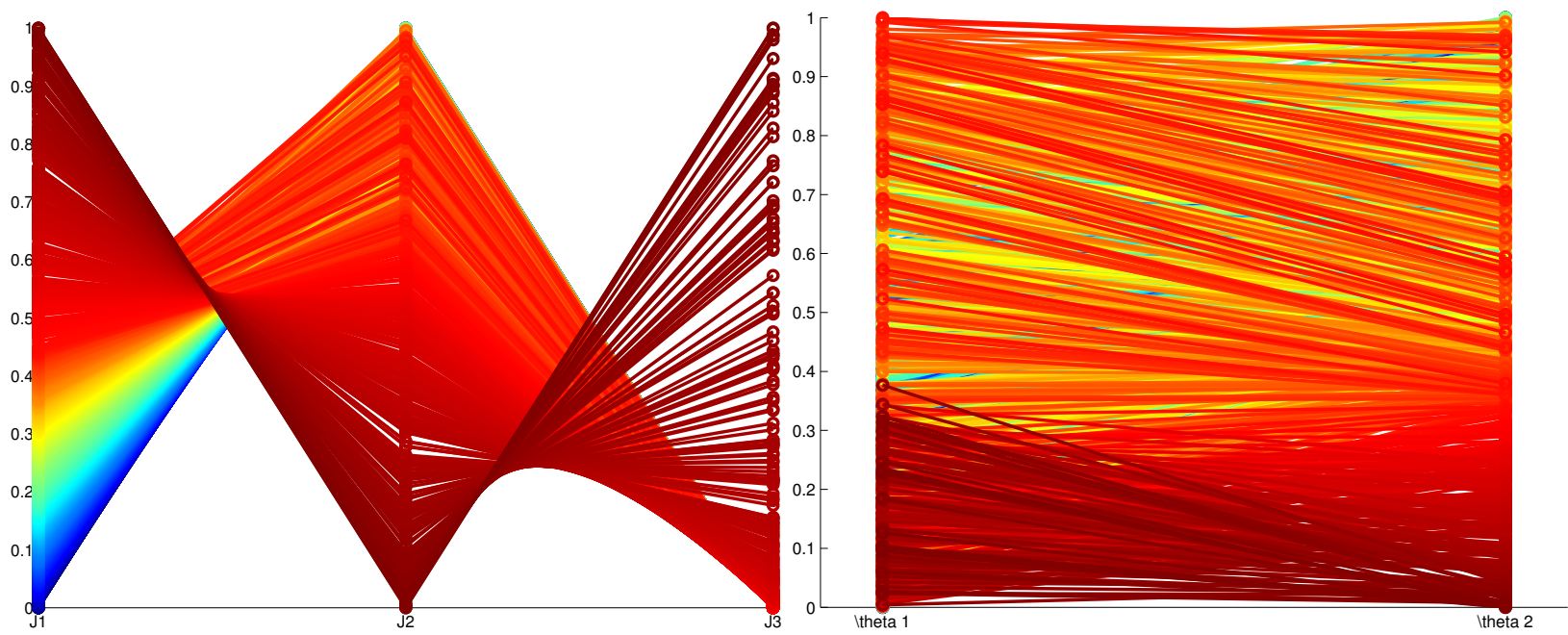

Figure 4: Parallel coordinate visualization for a Pareto front. Three objectives ([J1, J2,J3]) and two decision parameters $([\theta 1, \theta 2])$. y-axis have been normalized on objective and parameter range. 
The LD tool displays a two-dimensional graph for each objective and decision variable.

The ordered pairs $\left(J_{i}(\boldsymbol{\theta}),\|\hat{\boldsymbol{J}}(\boldsymbol{\theta})\|_{p}\right)$ in each objective sub-graph and $\left(\theta_{l},\|\hat{\boldsymbol{J}}(\boldsymbol{\theta})\|_{p}\right)$ in each decision variable sub-graph are plotted (a total of $n+m$ plots). Therefore, a given solution will have the same $y$ axis value in all graphs. This correspondence will help to evaluate general tendencies along the Pareto front and to compare solutions according to the selected norm. Also, with this correspondence, information from the objective space is directly embedded in the decision space, since a decision vector inherits its $y$-value from its corresponding objective vector, increasing completeness.

Level diagram visualization technique is not the only available option for Pareto front and set analysis, but it is recognized as easy to implement and with good properties [34. Other classical alternatives widely used are Parallel coordinates and Scatter plot, but the development of visualization techniques is constantly improving for n-dimensional problems, being the recent alternatives revised at [34. In order to compare Level diagram visualization with probably the most widely used alternative for Pareto analysis, Parallel plots, Figures 3 and 4 are supplied. The colors used in all these figures are only to identify the different points of the Pareto front and set (each point of the Pareto set has the same color at every plot) helping the interpretation and the comparison. Additionally, because the example has only three dimension at the objective space (and 2 dimension at decision space) the 3D plot and 2D plots are shown at Figure 2. Part of the following developments, the coloring techniques, can be used with differ visualization techniques, but there is a particular development (composed norm) that is specially suited for Level diagram representation. Then Level diagrams have been selected as the visualization technique for this article.

\section{Asymmetric norms assisting multi-dimensional visualization}

In this section we will develop the idea already introduced in Section 2.2 of the fact that the asymmetric norm allows to quantify the dominance relation of one solution to others for a given optimization problem. Therefore, asymmetric norm provides an easy and geometrically adapted tool for analyzing the domination relations between sets of points of $\mathbb{R}^{n}$. Let us show this step by step.

For a given pair of elements $J^{1}, J^{2} \in \mathbb{R}^{n}, d\left(J^{2}, J^{1}\right)=\left\|\left(J^{1}-J^{2}\right) \vee 0\right\|$ has a direct geometric meaning: how much is necessary to move $J^{1}$ to dominate $J^{2}$. In case we have $d\left(J^{2}, J^{1}\right)=0$, it means that $J^{1}$ already dominates $J^{2}$. When $d\left(J^{2}, J^{1}\right) \neq 0$, it supplies the distance projected into the subspace formed by the non-dominated dimensions. Figures 5 and 6, shows this basic interpretation for $2 \mathrm{D}$ and $3 \mathrm{D}$ spaces respectively. For instance, in 5 (a) the point $J^{1}$ has to be moved in both direction/dimension: $d_{1}$ in the objective $J_{1}$ and $d_{2}$ in the objective $J_{2}$, but in 5 (b) point $J^{1}$ only requires a displacement of $d_{2}$ in the objective $J_{2}$. The same interpretation could be done for 3D spaces (Figure 6) and high-dimensional spaces.

This useful property gives the possibility to add a new way to assist the DM when using graphical tools. Asymmetric norms could be used to color points of the Pareto front (and set) or to show relative displacement between points that are needed to change the dominance relation. 


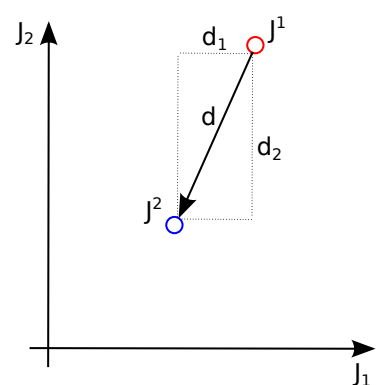

(a)

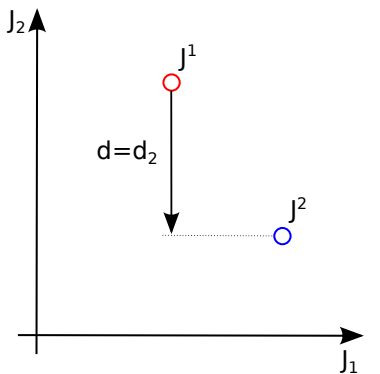

(b)

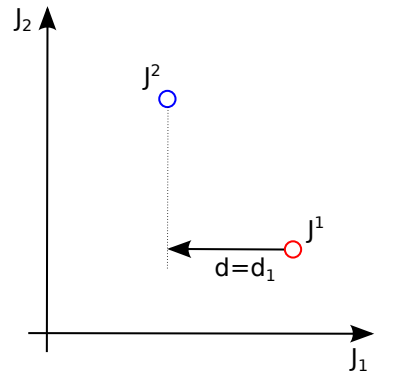

(c)

Figure 5: $d\left(J^{2}, J^{1}\right)$ in a 2D space for different cases. How much is necessary to move $J^{1}$ to dominate $J^{2}$ ?

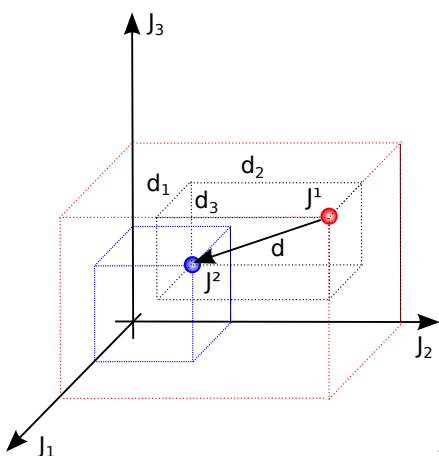

(a)

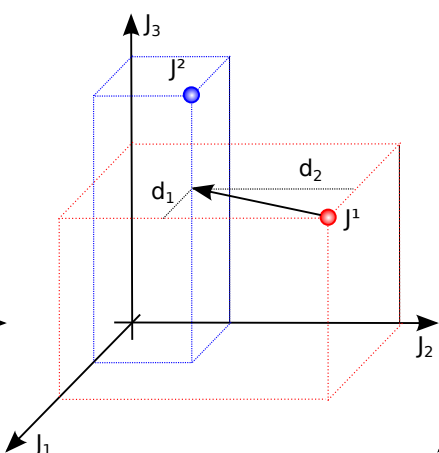

(b)

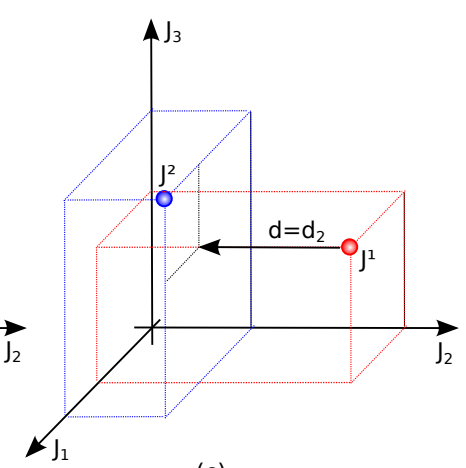

(c)

Figure 6: $d\left(J^{2}, J^{1}\right)$ in a 3D space for different cases. How much is necessary to move $J^{1}$ to dominate $J^{2}$ ? 
It is important to notice that the asymmetric distance gathers the dominance property and the Euclidean distance together. For the DM, both relations could be relevant. For some degradation in one or both objectives. To obtain more details the reverse asymmetric norms can be easily computed $d\left(J^{B}, J^{R}\right)=d 2$ and $d\left(J^{A}, J^{R}\right)=d 3$ showing than $J^{R}$ does not dominate none of the points and the distances in the projected non-dominated objectives.

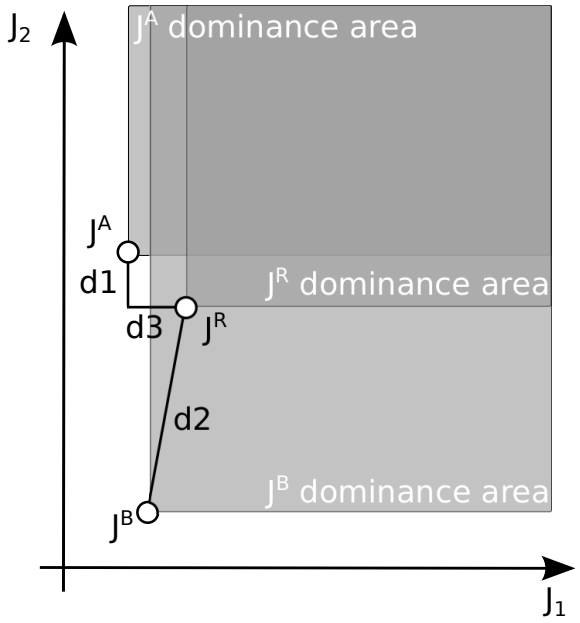

Figure 7: An example where a point $J^{A}$ is nearest to reference point $J^{R}$ than $J^{B}$, but point $J^{B}$ dominates $J^{R}$.

For a 2D space it is easy to see graphically when a point dominates other ones or how far Anyway, new computational methods to highlight dominated point and with the possibility to color according to relative distance to domination, are welcome.

For n-dimensional spaces it is necessary to use a specific visualization tool. Several visualization tools are available for designers to perform a multicriteria analysis [34]. The selection of one over another will rely on designer's preferences and willing to use a given tool. Here, in what it follows, we develop three applications of this asymmetric setting using the Level Diagrams framework for n-dimensional visualization. 
The characteristics of the asymmetric norm can also be seen with the LD representation. Figure 8 shows the same relations than figure 6 but with a Level Diagram visualization using to see for each dimension because the x-axis is in objective units. From one specific point, every point at its left side dominate it in this dimension/objective (but not necessarily in all the others).
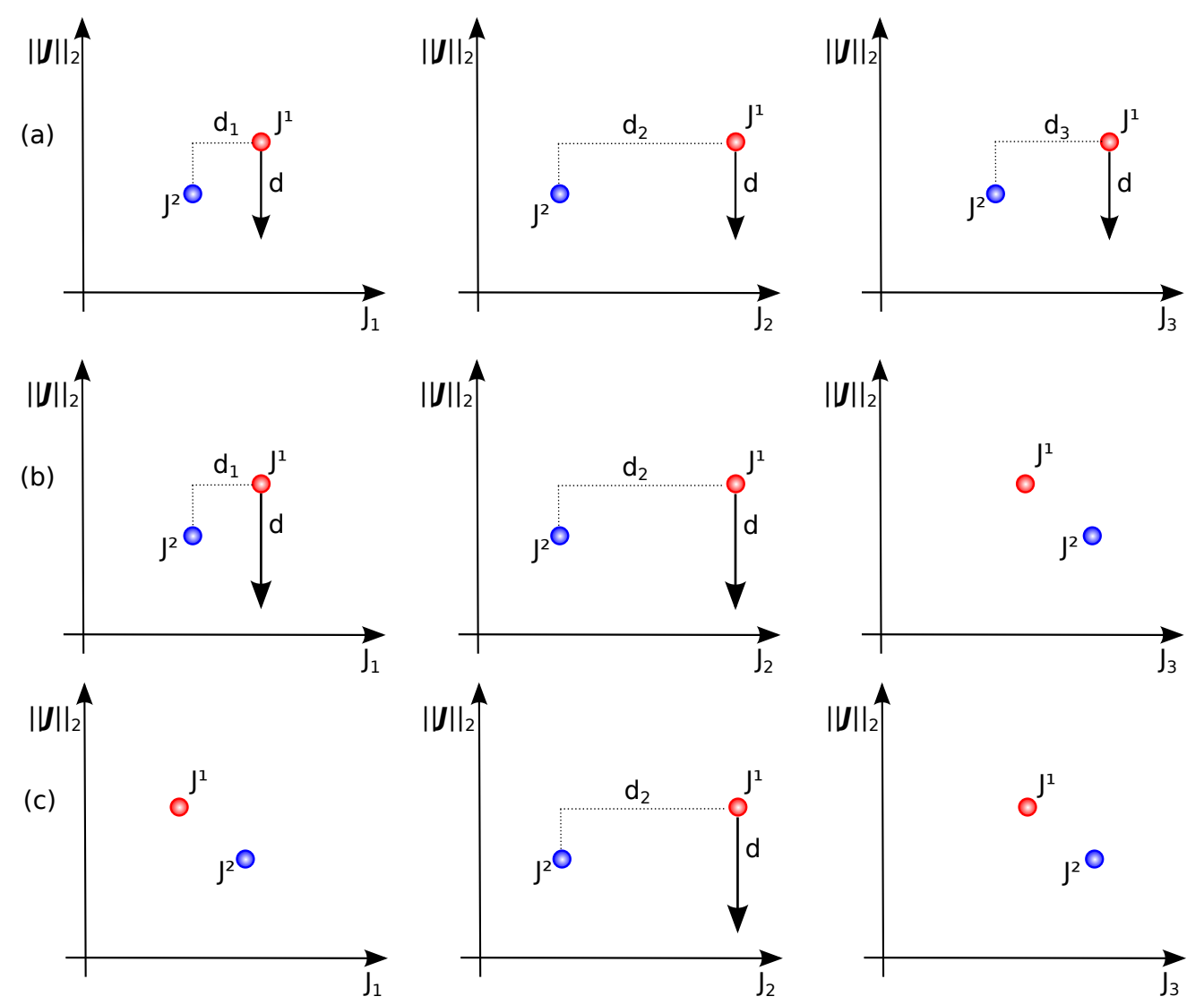

Figure 8: Asymmetric norm interpretation in a Level Diagram visualization. Correspond to cases in figure 6

\subsection{Coloring procedure based on asymmetric norms for Pareto front and set graphical rep- resentation}

In this section we propose an asymmetric distance based rule for helping the DM to select one of the optimal solutions. This proposal is oriented to MOP with more than two dimensions.

When the designer chooses a desired point, it is possible to use the asymmetric distance to classify every point of the Pareto Front. The value of this distance shows to the designer how much the Pareto front point are close to her/his preferences. This classification can be used to color the Pareto front points according to it and can help in a graphical interpretation. 
After obtaining an approximation of the Pareto set $\Theta_{P}^{*}$ (and its corresponding Pareto front $\left.\boldsymbol{J}_{P}^{*}\right)$, the DM chooses a point which summarizes what under her/his criteria is an optimal

solution $J^{0} \in \mathbb{R}^{n}$. The DM does not know a priory if $J^{0} \in \boldsymbol{J}_{P}^{*}$ (typically $J^{0} \notin \boldsymbol{J}_{P}^{*}$ ).

The scheme of this procedure requires the next steps.

(1) Computing the asymmetric distance between $J^{0}$ and every $J \in \boldsymbol{J}_{P}^{*}$,

$$
d\left(J^{0}, J\right)=\left\|\left(J-J^{0}\right) \vee 0\right\|
$$

(2) Color assignation according to asymmetric distance. Let $C$ be the set of colors, that is parametrized as 3-coordinates positive vectors (e.g. RGB values). We define a predetermined continuous function $f: \mathbb{R}^{+} \rightarrow C$ in such a way that the value $f(0)$ represents the best approximation color (in the following example this color is blue), and the color is changing as the value of $f$ is increasing (for example, from black to white using the Matlab hot colormap). Consider the function $g: R^{n} \rightarrow C$ that is defined by composing $f$ and $d$ as $g(J):=f\left(d\left(J^{0}, J\right)\right)$, i.e. to each element $J \in \boldsymbol{J}_{P}^{*}$ we assign the color of $C$ that is given by $g(J)$. The same colors are used for the level diagrams that represent the Pareto set approximation $\Theta_{P}^{*}$.

Using level diagrams to represent the Pareto set approximation $\Theta_{P}^{*}$ and its corresponding Pareto Front $\boldsymbol{J}_{P}^{*}$. The result is the following:

(a) All the elements of $\boldsymbol{J}_{P}^{*}$ that dominate $J^{0}$ appear in blue. All of them are better than the original expectation of the DM, who can choose the better one among them following her/his criterion. Remark that the rest of the points are also colored according to the colormap used.

(b) There are no elements appearing in blue; the DM must choose among those ones that have the darkest color (closer to dominate $J^{0}$ ).

\subsection{Coloring Pareto solutions according to a preference interval criterion}

A second alternative where the asymmetric norm may be used in order to assist the graphical interpretation in MCDM is when the DM has a clear idea of how to classify the different solution in a scale, e.g. from "Highly Desirable" solutions to "Highly Undesirable" ones. A possible classification (but not the only one) could be: "Highly Desirable" (HD), "Desirable" (D), "Tolerable" (T), "Undesirable" (U) and "Highly Undesirable" (HU).

Suppose that the DM is able to divide the domain of each coordinate $J_{i}$ (objective) in intervals $\left.\left.\left.] I_{i}^{0}, I_{i}^{1}\right], \cdots,\right] I_{i}^{k-1}, I_{i}^{k}\right], i=1, \ldots, n$ and $k \in \mathbb{N}$ (the number of intervals) corresponding to the above scale. In such case, the DM has to fulfill the values of a table similar to the one of Table 1. Remark that the values of this table are expressed in the objective units, where usually the DM is more familiar with it.

Let us write $P_{r}$ for each hypercube defined in such a way that its vertex with bigger norm of the hypercube (the one that is dominated by the rest of the hypercube) correspond to 


\begin{tabular}{|c|c|c|c|c|c|c|c|c|c|c|c|}
\hline $\begin{array}{l}\text { Labels } \\
\text { Interval limits }\end{array}$ & & $\overleftarrow{I_{i}^{o}} \quad \mathrm{HD}$ & $\begin{array}{l}\rightarrow \leftarrow \\
I_{i}^{1}\end{array}$ & $\mathrm{D}$ & $\begin{array}{l}\rightarrow \leftarrow \\
I_{i}^{2}\end{array}$ & $\mathrm{~T}$ & $\begin{array}{r}\rightarrow \leftarrow \\
I_{i}^{3}\end{array}$ & $\mathrm{U}$ & $\begin{array}{l}\rightarrow \leftarrow \\
I_{i}^{4}\end{array}$ & $\mathrm{HU}$ & $\overrightarrow{I_{i}^{5}}$ \\
\hline Objective 1 & $J_{1}$ & - & - & & - & & - & & - & & - \\
\hline Objective 2 & $J_{2}$ & - & - & & - & & - & & - & & - \\
\hline & $\ldots$ & $\ldots$ & $\ldots$ & & $\ldots$ & & $\ldots$ & & $\ldots$ & & $\ldots$ \\
\hline Objective $\mathrm{n}$ & $J_{n}$ & - & - & & - & & - & & - & & - \\
\hline
\end{tabular}

Table 1: Typical table for preference intervals in a MOP.

$I^{r}=\left(I_{1}^{r}, \ldots, I_{n}^{r}\right)$ (the points defined by the columns of table 1). The asymmetric distance from any point $J$ to a vertex $I^{r}$ gives easily in which hypercubes $J$ is located.

$$
P_{r}=\left\{J \in \mathbb{R}^{n}: d\left(I^{r}, J\right)=0\right\}
$$

These hypercubes enclose the volume where all the objectives are limited by the values of $I^{r}$. Beginning from the smaller one $P_{1}$, each hypercube is included inside the next ones: $P_{1} \subset P_{2} \subset \cdots \subset P_{k}$. For the DM it is worthwhile to quickly localize in which hypercube $r$ a point of the Pareto front (and set) is placed, in particular the smaller $P_{r}$.

The procedure colors according to the membership of each point to one of these hypercubes is established as follows:

(1) Computing hypercube membership. We define $g: \mathbb{R}^{n} \rightarrow \mathbb{N}$ as a function that gives which is the smaller hypercube where a point of the objective space $J$ is located (according to a particular range of preferences).

$$
g(J):=\min _{r}\left\{r: d\left(I^{r}, J\right)=0\right\}=\min _{r}\left\{r: J \in P_{r}\right\} .
$$

For each solution of the Pareto front $J \in \boldsymbol{J}_{\boldsymbol{P}}^{*}$ the hypercube is computed as $g(J)$.

(2) Assigning color according to hypercubes. Let $C$ be the set of colors, that is parametrized as 3-coordinates positive vectors (RGB values). We define a predetermined continuous function $f: \mathbb{N} \rightarrow C$. The color assigned to each Pareto point $\boldsymbol{\theta} \in \Theta_{P}^{*}$ and $J=\boldsymbol{J}(\boldsymbol{\theta}) \in \boldsymbol{J}_{\boldsymbol{P}}^{*}$ (for both, objective space and solution space representation) is computed as: $c=f(g(J))$

\subsection{Composed norm based on preferences for level diagram syncrhonization}

Alternatively or additionally to the described coloring procedure based on preference ranges, it can be useful for the DM to localize visually the points of the Pareto front and set that are in each interval of the preference matrix. For a 2-dimensional Pareto front it is easy to see (grayed areas in scatter plot at Figure 9), but for problems with more dimensions the visualization technique have difficulties to reproduce the hypercubes corresponding to each interval of preferences. Figure 9 shows a comparison between scatter representation and level diagram for a 2D Pareto front. For higher dimension the visualization of hypercube is not 

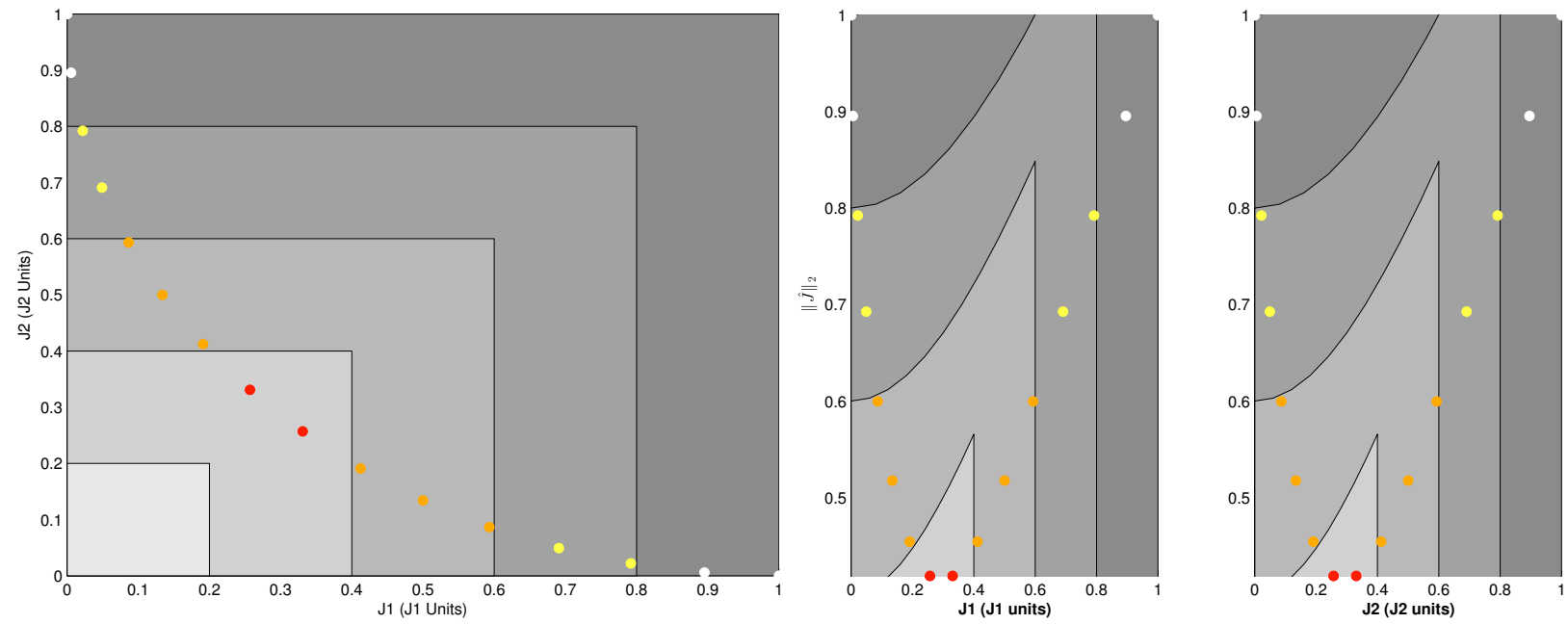

Figure 9: Hypercubes visualization in scatter plot and level diagram

clear because the bounds of each hypercube are hyperplanes and produce cloud of points in level diagrams.

With the LD representation it is possible to use different norms for the synchronization of the y-axis. The default choice is to use the euclidean norm $\|\hat{\boldsymbol{J}}(\boldsymbol{\theta})\|_{2}$ but a composed norm based on asymmetric distance $\|\hat{\boldsymbol{J}}(\boldsymbol{\theta})\|_{c n}$ can layer each point according to the preference interval and produce a way to visualize the hypercube membership corresponding to each interval. This might improve the persistence and completeness of this visualization, concerning the preferability hypercube of a given solution. Additionally a coloring procedure could be used to show other type of information rather than showing hypercube membership.

The proposal is to use the vertexes of each hypercube (corresponding to each preference interval) to calculate the asymmetric norms. Beginning with the least preferred interval ("Highly Undesirable"), the points with a non-zero value of the asymmetric norm doesn't dominate the vertex and consequently they are outside of the "Highly Undesirable" hypercube. Additionally the value of its asymmetric norm shows the euclidean distance (in the non-dominated subspace) to the hypercube; in fact it shows the distance to dominate the vertex of the hypercube. This value could be used for the classification of each point.

The points with zero asymmetric norm pass to the next steps consisting in repeating the process for the following hypercubes, each step with the hypercube of the next preference interval. At the end of this calculation procedure, each point has a value assigned that indicates the euclidean distance (in the non-dominated subspace) to the next non-dominated hypercube.

To represent all these distance values in the same axis, an offset value for each hypercube is required to show the different hypercube membership. Several alternatives can be possible and the proposal for this offset is to use the sum of the highest asymmetric distances for each one of the previous hypercubes.

The calculated value (composed norm) $\|J\|_{c n}$ is used for y-axis synchronization among 
level diagram representation. Figure 10 shows an example of a level diagram synchronized by the composed norm. In this example the offset applied for each hypercube is: zero for Desirable hypercube, $d_{4}$ for Tolerable hypercube and $d_{1}+d_{4}$ for Undesirable hypercube. The composed norm is computed with the offset of the corresponding point plus the asymmetric distance of the point to the next hypercube vertex.
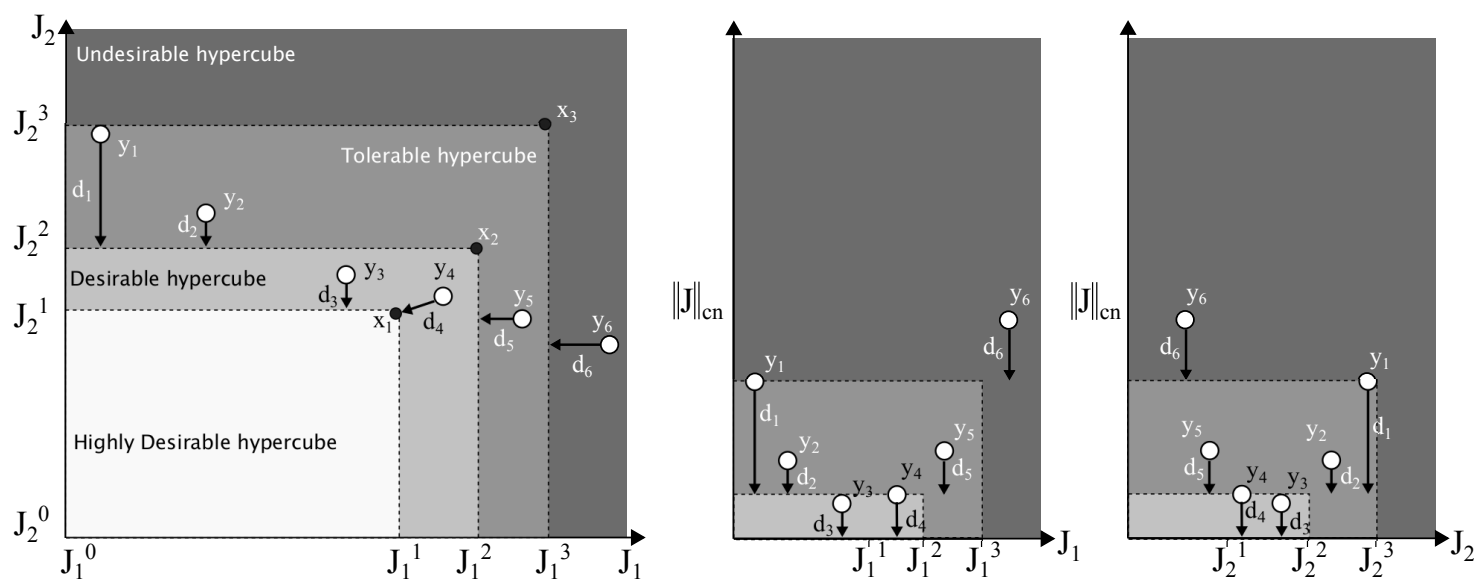

Figure 10: Components of the composed norm

This procedure to obtain the composed norm can be detailed as:

(4) Consider the constants $M_{x}:=\max \left\{\phi(J): J \in A_{x}\right\}$

(5) Finally, for each $J \in A_{x}$, define the composed norm $\|J\|_{c n}$ as

$$
\|J\|_{c n}:=\phi(J)+\sum_{x \leq x_{o}} M_{x}
$$

Algorithm 1 1 shows a pseudocode to obtain the composed norm.

where we assume that $I^{0}=\emptyset$.

(3) Define the function $\phi$ as follows: if $J \in A_{x}$ then $\phi(J):=d\left(J, I^{x-1}\right)$. 
Data: $J_{p}^{*}$ (Pareto Front approximation), $I(0) \cdots I(r)$ (Table of preferences)

Result: Jcn (Composed Norm)

for each $p(i) \in J_{p}^{*}$ do

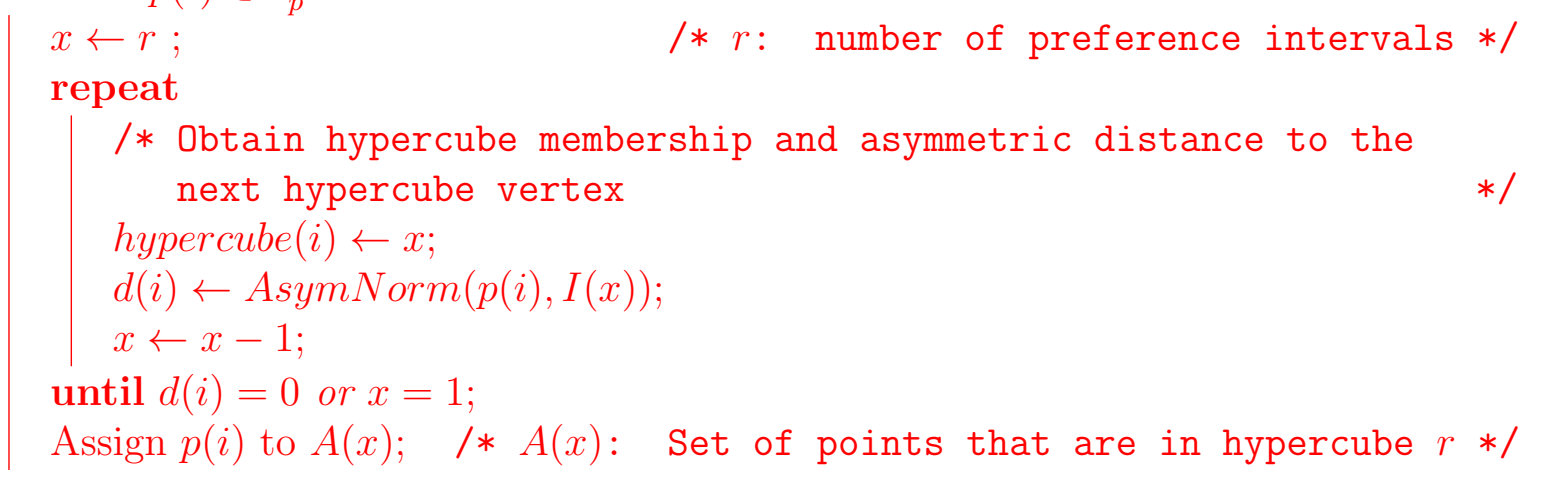
end

for $x \leftarrow 1$ to $r$ do

/* Obtain maximum distance inside each $\mathrm{A}(\mathrm{x})$ and compute offset */ $d \max (x) \leftarrow \max (d(i), \forall p(i) \in A(x))$;

if $x=1$ then

off $\operatorname{set}(x) \leftarrow 0$

else

$\mid \operatorname{off} \operatorname{set}(x) \leftarrow$ offset $(x-1)+\operatorname{dmax}(x-1)$

end

end

for each $p(i) \in J_{p}^{*}$ do

/* Update asymmetric distance with corresponding offset */

$J c n(i) \leftarrow d(i)+$ off set $($ hypercube $(i))$;

end

Algorithm 1: Pseudocode for composed norm $\|J\|_{c n}$ 


\section{Examples}

In this section, three examples are analyzed, they show analysis on 2, 5 and 6 dimensions for the objective vector space. The first example is used to show that no information (completeness) or clarity (simplicity) will be lost using the LD instead a 2-D graphical analysis. The two latter case depict the visualization capacity of LD using asymmetric norms.

For all examples, the Pareto fronts are calculated using multiobjective algorithms as spMODEII [29] or evMOGA [23]), but any other algorithm or procedure could be used since we are developing tools for the MCDM step.

\subsection{Bi-objective truss design problem}

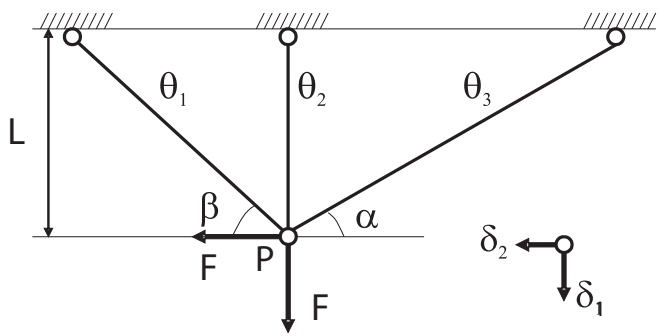

Figure 11: Truss structure to optimize as a 2D MOP.

Whereas it could be easiest (from a persistence point of view) to use a straightforward approach with the Scatter Plot visualization, this example is included and used to cover the following topics:

- Bring a first approach to the LD visualization colored with the use of asymmetric distance.

- Introduce composed norm for LD visualization.

The truss design problem is a classical MOO benchmark statement to test algorithms, as well as decision-making step procedures. The three-bar truss parameters proposed in [25] and [32] are used, that means the design variables correspond to the sections of the bars $\theta=\left[\theta_{1}, \theta_{2}, \theta_{3}\right]$ see Figure 11. Two objectives are minimized: deflection or displacement of node $\mathrm{P}, J_{1}(\boldsymbol{\theta})=0.75 \delta_{1}+0.25 \delta_{2}$ (in $\mathrm{cm}$ ), and total volume $J_{2}(\boldsymbol{\theta})$ (in $\mathrm{cm}^{3}$ ). Additionally some constraints have to be fulfilled, the stresses in each bar are limited to 200M Pa.

As a first example, following the procedure described in section 3.1 it is supposed that the designer $(\mathrm{DM})$ has an approximation to the Pareto set $\boldsymbol{\Theta}_{\boldsymbol{P}}^{*}$ and its corresponding Pareto front $\boldsymbol{J}_{P}^{*}$ (whatever the optimization algorithm has been used). For the example of the three bar truss a Pareto set of 48 solutions is available. For this Pareto set a Pareto front is associated with it and have to supply valuable information for the final choice. For the first demonstration it is supposed that the DM knows a point that can summarize her/his criteria for a pretended optimal solution: $J^{0}=\left[0.12 \mathrm{~cm}, 400 \mathrm{~cm}^{3}\right]$. 

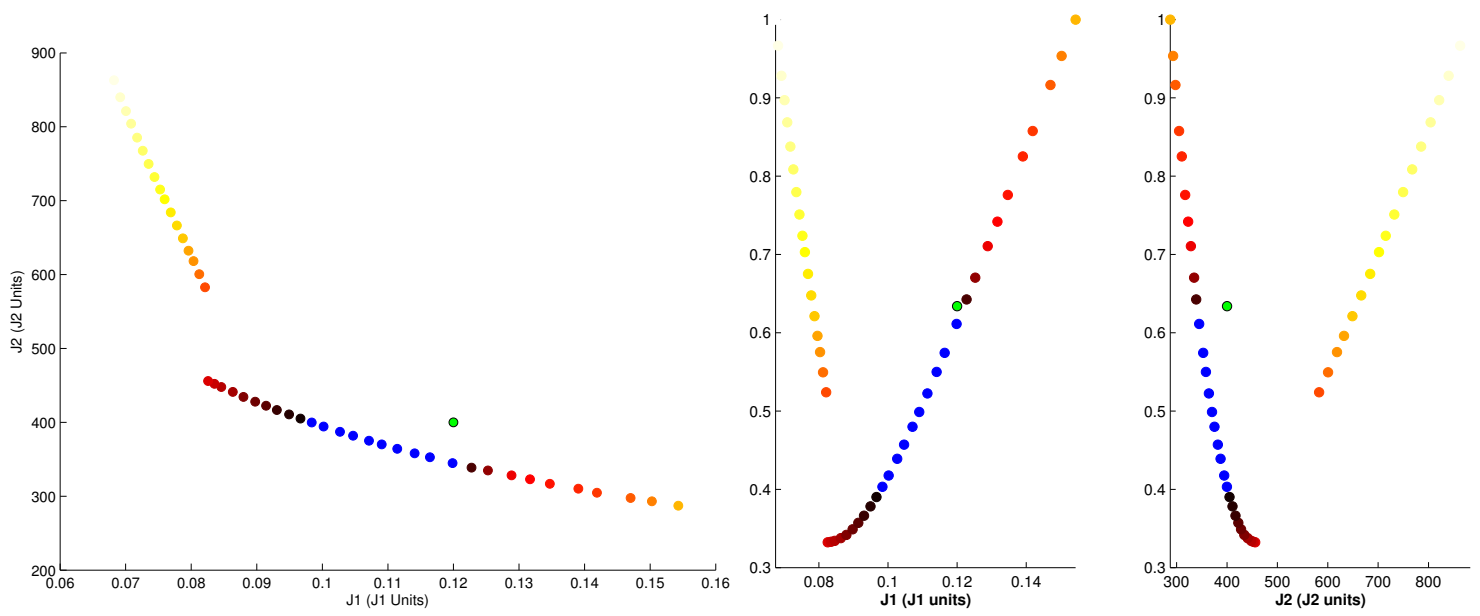

Figure 12: Colored Pareto front according to asymmetric distance with normalization of objectives. 2D representation on the left and level diagram representation on the right. Blue points have a zero asymmetric distance to the $J^{0}$ point (in green), for the rest (non-zero asymmetric distance) the darker the the shorter the distance.

Before the next step of the procedure, the Pareto front is normalized according to the range of values of the Pareto front. That indicates that the full ranges of variation of each objective are equally important. Of course this normalization is subjective and depends on the DM preferences. In the example the available Pareto front is in ranges: $J_{1} \in[0.0675,0.1543] \mathrm{cm}, J_{2} \in[287.2,882.8] \mathrm{cm}^{3}$. The resulting normalized objectives:

$$
J n_{1}=\frac{J_{1}-0.0675}{0.1543-0.0675} ; J n_{2}=\frac{J_{2}-287.2}{882.8-287.2}
$$

After this selection, the asymmetric distance between every point of $\boldsymbol{J}_{P}^{*}$ and $J^{0}$ is computed and a color is assigned to every point of $\boldsymbol{J}_{P}^{*}$. Blue color is assigned to the points with asymmetric distance of zero. For the points with a non-zero distance, the darker color indicates a lower distance and the lighter color a higher distance. For comparison purpose figure 12 shows both the 2D classical representation and the level diagram representation the $J^{0}$ point is shown in green.

For a bi-dimensional problem Level Diagram is not as intuitive as a 2D scatter plot but the same information is depicted. The $\mathrm{x}$-axes of the level diagrams shows the objective values, then it is easy to see that for a particular point (for instance $J^{0}$ ) the points on its left have a better value. The blue points (the ones with zero asymmetric norm and then that dominate $J^{0}$ ) are on the left of $J^{0}$ in both objectives. The darker points (lower asymmetric distance) are closer to dominate $J^{0}$ than the lighter points.

For the next demonstration a range of preferences has to be established. In [32] several scenarios are defined by the DM, for demonstration purpose only one of them will be used. In particular the DM has selected 5 range of preferences see table 2 Highly Desirable (HD), Desirable (D), Tolerable (T), Undesirable (U), Highly Undesirable (HU). 


\begin{tabular}{|c|c|c|c|c|c|c|c|c|c|c|c|}
\hline & & $\overleftarrow{I_{i}^{o}}$ & $\mathrm{HD}$ & $\begin{array}{l}\rightarrow \leftarrow \\
I_{i}^{1}\end{array}$ & $\mathrm{D}$ & $\begin{array}{l}\rightarrow \leftarrow \\
I_{i}^{2}\end{array}$ & $\mathrm{~T}$ & $\begin{array}{l}\rightarrow \leftarrow \\
I_{i}^{3}\end{array}$ & $\mathrm{U}$ & $\rightarrow \underset{I_{i}^{4}}{\leftarrow} \mathrm{HU}$ & $\overrightarrow{I_{i}^{5}}$ \\
\hline Deflection $(\mathrm{cm})$ & $J_{1}$ & 0.0 & & 0.10 & & 0.12 & & 0.14 & & 0.16 & 0.20 \\
\hline Volume $\left(\mathrm{cm}^{3}\right)$ & $J_{2}$ & 200 & & 300 & & 500 & & 600 & & 900 & 1200 \\
\hline
\end{tabular}

Table 2: Preference range for three-bars truss MOP.

According to this table, it is easy to obtain the hypercube where each point of the Pareto front is placed following procedure of section 3.2. Remark that the vertexes correspond to the points supplied by the DM's table of preferences.

For a 2D problem it is not strictly necessary coloring the Pareto point to see to which range they belongs. It is enough to plot the area for each hypercube and inspect it visually. The scatter plot on the left at Figure 13 shows these areas with different levels of gray. For higher dimensional problems, the visualization techniques have difficulties to show these areas/hypercubes. Therefore for these type of representation an alternative has to be stated. So far, two proposals based on asymmetric norm have been presented, the first one is based on coloring the points of the Pareto front according the hypercube where they are placed (Section 3.2 ). The second alternative consists on the use of a composed norm instead of euclidean norm to synchronize Level Diagrams y-axis (Section 3.3).

In the former case, the coloring methodology based on asymmetric norm helps to localize visually and easily where each solution is place. This analysis is easy to perform in scatter plot and Level diagrams. Additionally, in this example, it seems (comparing figures 13 and 14) that Level diagrams propagates better such information to the Pareto set (therefore, improving completeness).

In the latter case, in order to represent the hypercube membership a different alternative is used trying to depict the hypercube space in the Level diagram representation. In the case of level diagram (using euclidean distance on y-axis, $\left.\|\hat{\boldsymbol{J}}(\boldsymbol{\theta})\|_{2}\right)$, the representation of the grayed areas is not so easy. In a first step the coordinates of the hyperplanes that limits these hypercubes have to be normalized according to the normalization used for the Pareto front and later the norm used for y-axis have to be calculated $\left(\|\hat{\boldsymbol{J}}(\boldsymbol{\theta})\|_{2}\right)$. See the representation of the "Desirable" hypercube represented by points A, B and C in the Figure 14. Remark that the representation of each hypercube in Level diagrams for bi-objective problems is affordable (lines in scatter plot are converted in lines in level diagram) but the representation of hypercubes of 3 or more dimension is not so clear.

Using the proposed composed norm based on asymmetric distance for Level diagram synchronization, each Pareto front point is clearly positioned in one of the hypercubes and the Pareto set representation shows clearly the membership hypercube without the needing of any color and propagating even further this information, see Figure 15. Then, the color can be used to show other characteristics of the points. At Figure 15 it is used to better localize each point, a point has the same color at each diagram. 

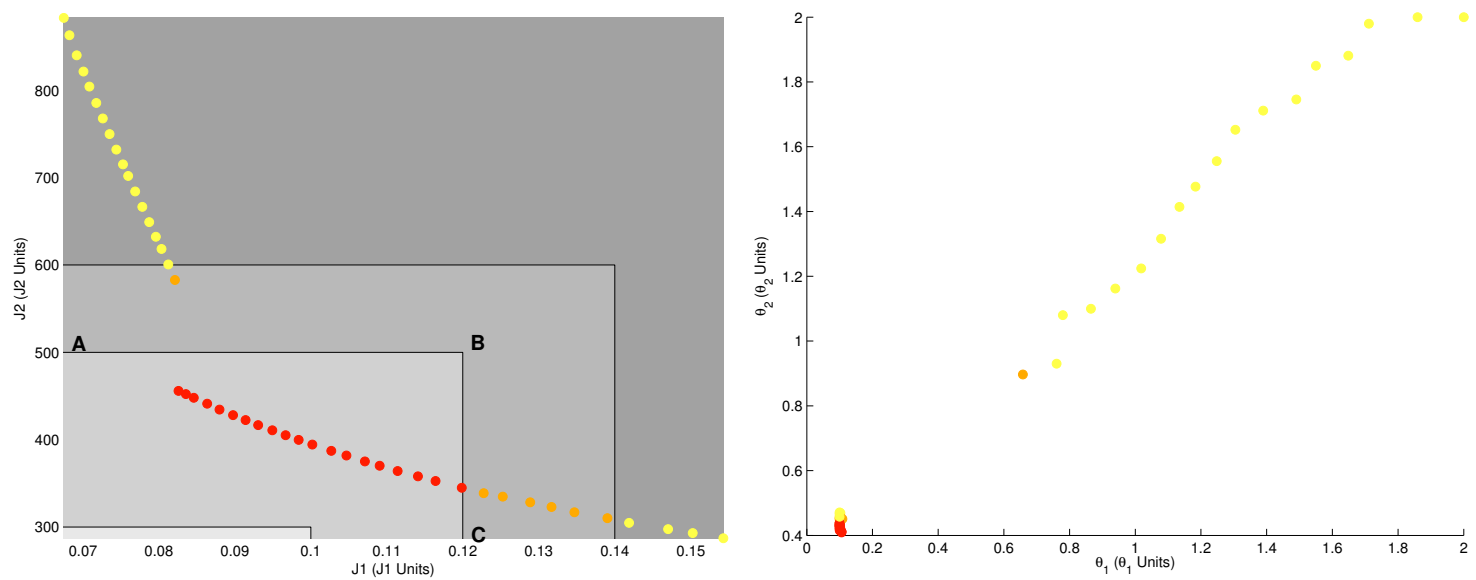

Figure 13: Colored Pareto front according to hypercube form preference range assisted by asymmetric distance. 2D Pareto front representation on the left and Pareto set representation on the right. Darker color (redder) means points on a better hypervolume. The ranges of preferences have been indicated with grayed areas (darder grey means worst range). "Desirable" hypercube corresponds to vertex A,B,C.
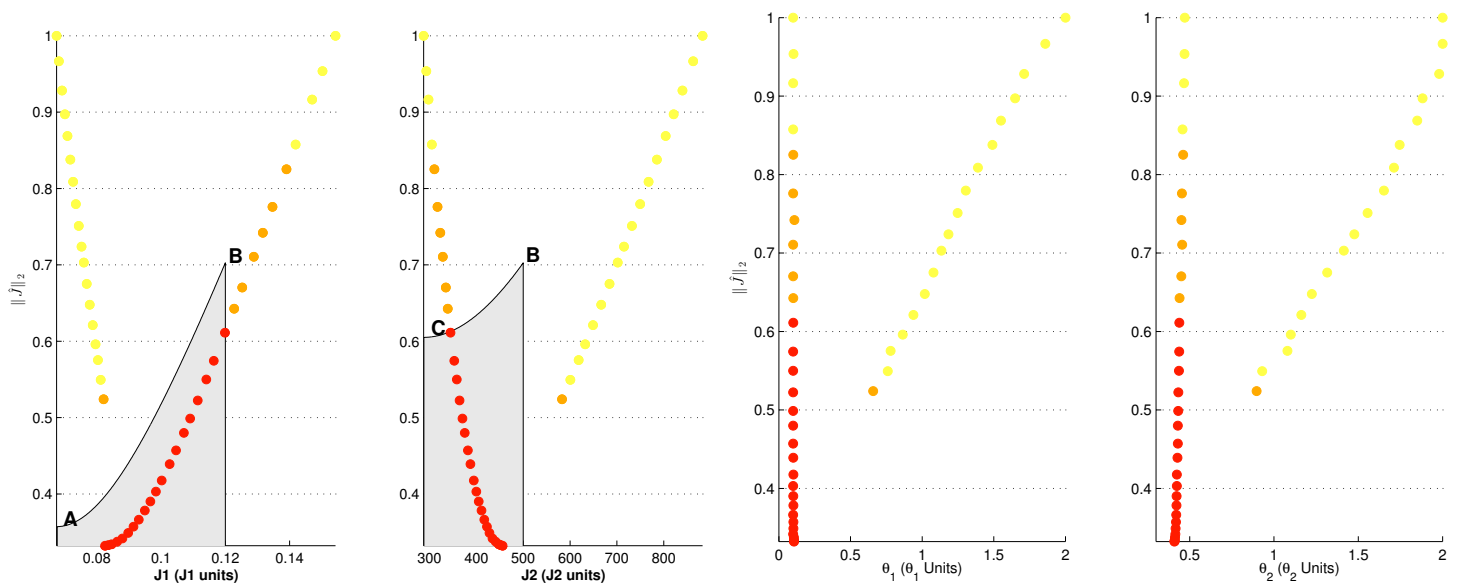

Figure 14: Colored Pareto front according to hypercube form preference range assisted by asymmetric distance. Level diagram with euclidean norm for y-axis synchronization. Pareto front the left and Pareto set on the right. Darker color (redder) means points on a better hypervolume. "Desirable" hypercube corresponds to vertex A,B,C. 

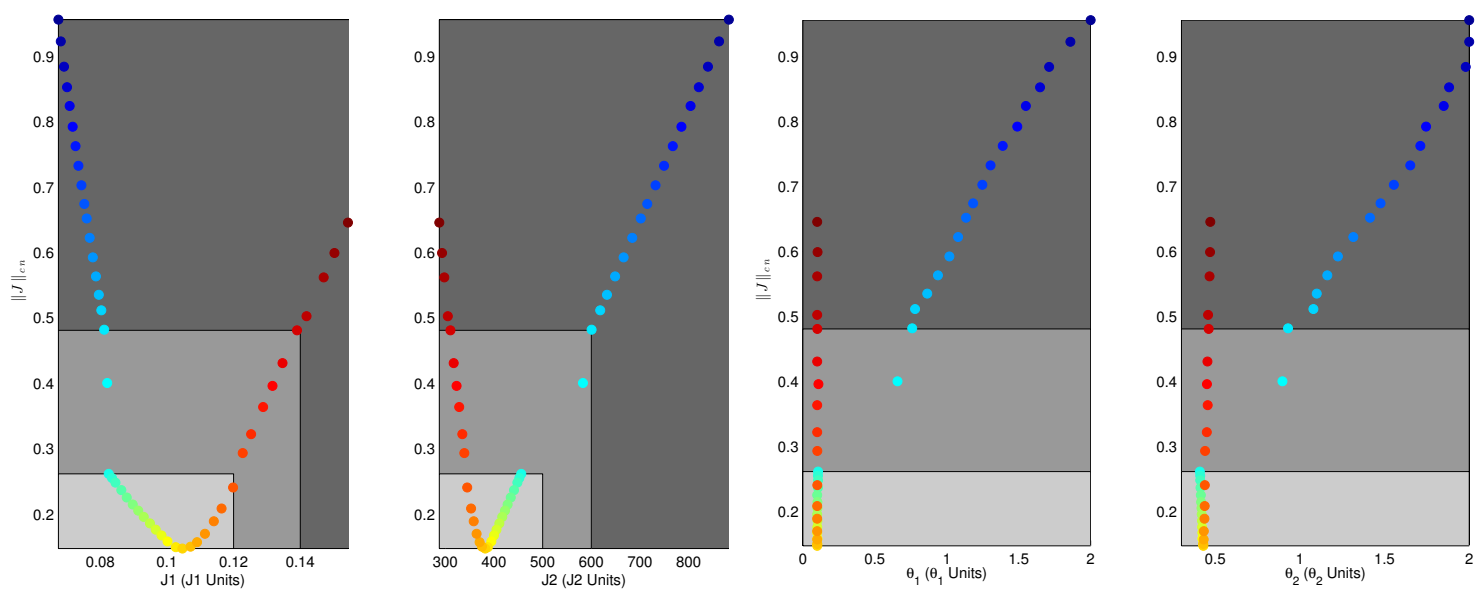

Figure 15: Level diagram with composed norm for y-axis synchronization. Pareto front the left and Pareto set on the right. The ranges of preferences (hypercubes) have been indicated with grayed areas (darker grey means worst range). Each point of the Pareto set and front have the same color at each diagram.

\subsection{Parametric controller design}

The next example is a parametric controller design for the control benchmark proposed at the American Control Conference (ACC) [35]. The MOP statement described in [3, 30] is used. The aim with this example is to demonstrate the viability of the approach with more than two objectives. It has six objectives to minimize: robust stability $\left(J_{1}(\boldsymbol{\theta})\right)$; maximum control effort $\left(J_{2}(\boldsymbol{\theta})\right)$, worst case settling time $\left(J_{3}(\boldsymbol{\theta})\right)$; noise sensitivity $\left(J_{4}(\boldsymbol{\theta})\right)$; nominal control effort $\left(J_{5}(\boldsymbol{\theta})\right)$; and nominal settling time $\left(J_{6}(\boldsymbol{\theta})\right)$.

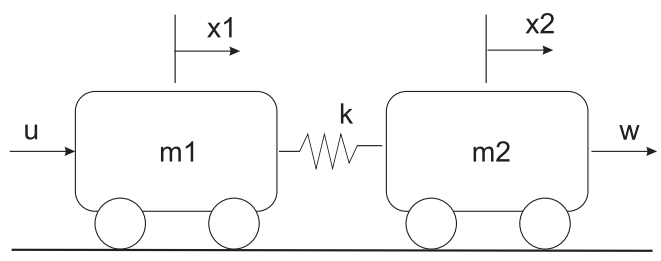

Figure 16: ACC benchmark problem used to test a 6-dimensional MOP.

Only one controller structure $G(s)$ will be evaluated:

$$
G(s)=\frac{\theta_{1} s^{2}+\theta_{2} s+\theta_{3}}{s^{3}+\theta_{4} s^{2}+\theta_{5} s+\theta_{6}}
$$

The limits for controller parameters are (search space): $-1 \leq \theta_{i} \leq 10$. To avoid extreme value that are not interesting for the $\mathrm{DM}$ the following constraints have been added to the problem. 


$$
\begin{array}{rll}
J_{1}(\boldsymbol{\theta}) \leq-0.0001 ; & J_{2}(\boldsymbol{\theta}) \leq 2 ; & J_{3}(\boldsymbol{\theta}) \leq 40 \\
J_{4}(\boldsymbol{\theta}) \leq 1.5 ; & J_{5}(\boldsymbol{\theta}) \leq 2 ; & J_{6}(\boldsymbol{\theta}) \leq 25
\end{array}
$$

An approximation of the Pareto set (and its associated Pareto front) of 1421 points is obtained using an evolutionary multiobjective algorithm. The decision making step is performed using LD and coloring methodologies assisted by asymmetric norms, as previously described. The first step in the following procedures is the normalization of the Pareto front and Figure 20 for Pareto set representation using $\|\hat{J}\|_{2}$ for y-axis synchronization. The coloring methodology shows quickly the preference interval where each solution is placed in the objective and parameter spaces. 

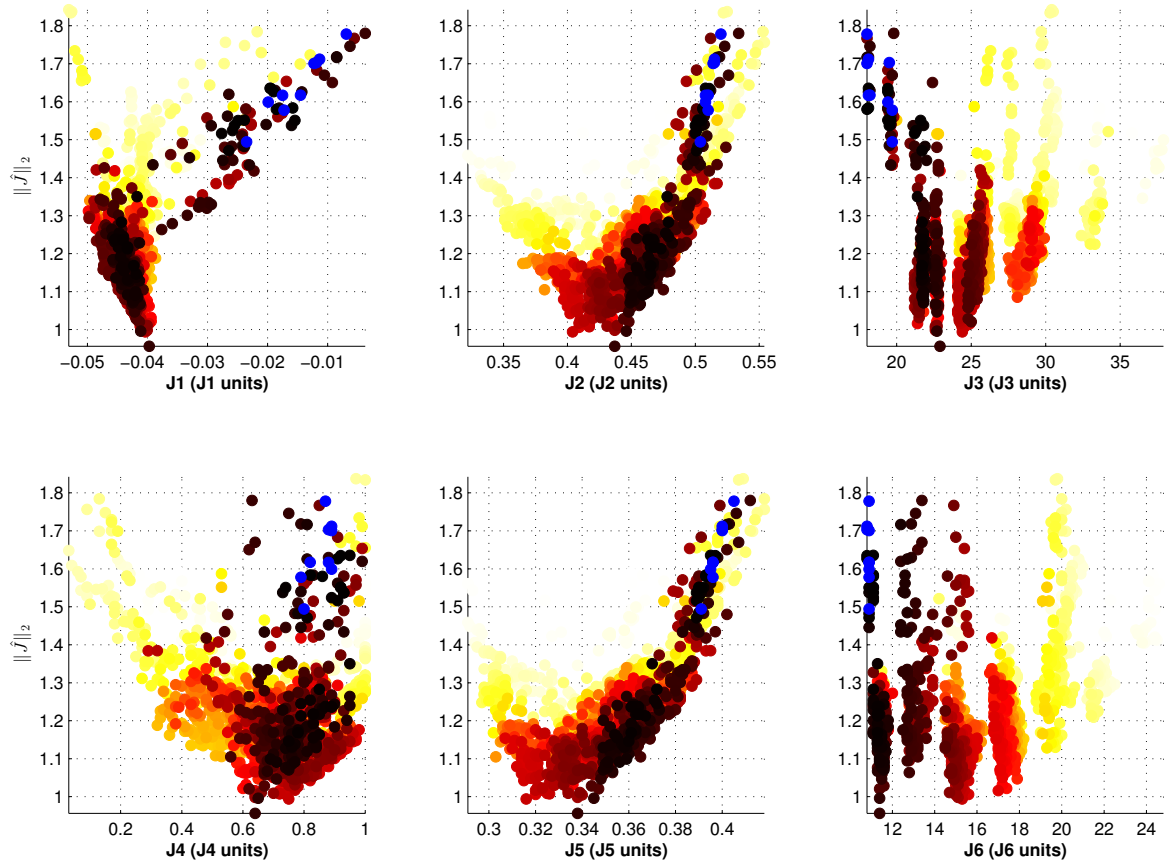

Figure 17: Level diagram representation of the Pareto Front for ACC benchmark problem. $\|\hat{J}\|_{2}$ for y-axis synchronization Colored with asymmetrics norms for tolerable point
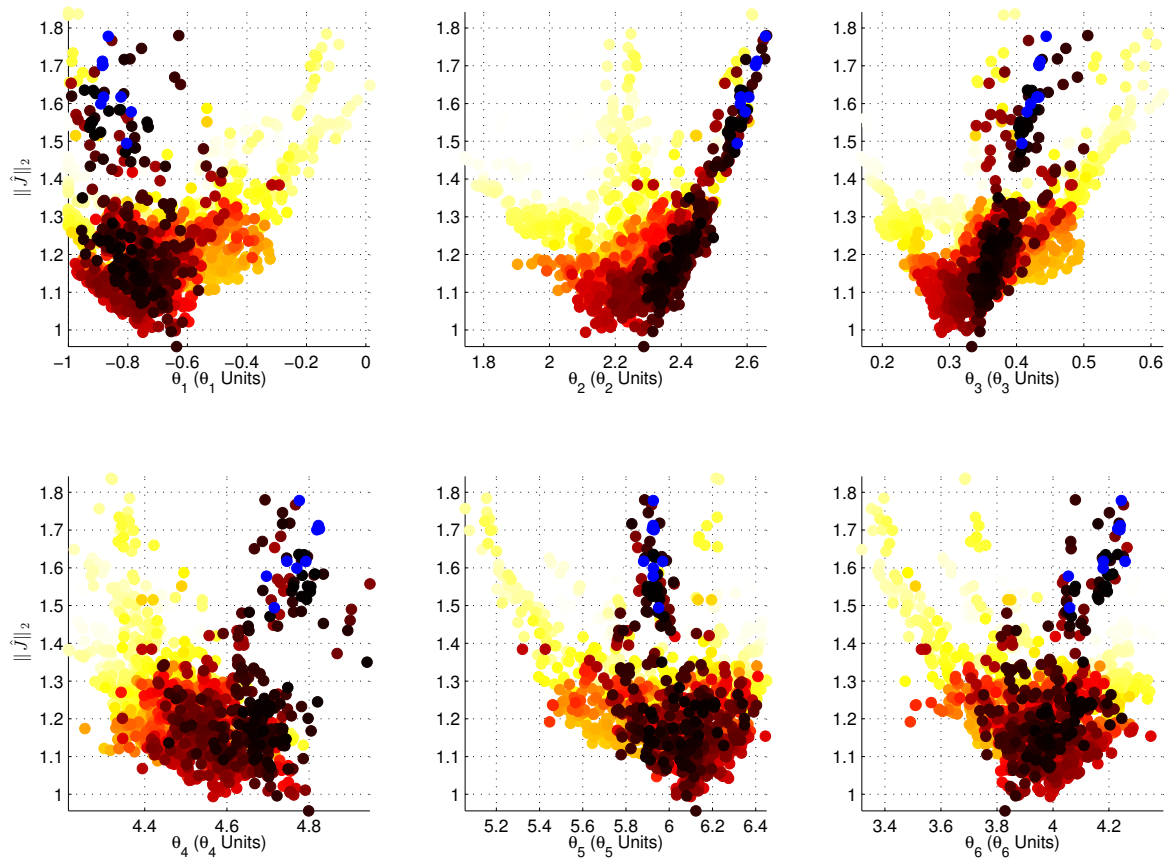

Figure 18: Level diagram representation of the Pareto Set for ACC benchmark problem. $\|\hat{J}\|_{2}$ for y-axis synchronization Colored with asymmetrics norms for tolerable point 


\begin{tabular}{|c|c|c|c|c|c|c|c|c|c|c|c|c|}
\hline & & $\overleftarrow{I_{i}^{o}}$ & $\mathrm{HD}$ & $\begin{array}{l}\rightarrow \leftarrow \\
I_{i}^{1}\end{array}$ & $\mathrm{D}$ & $\begin{array}{l}\rightarrow \leftarrow \\
I_{i}^{2}\end{array}$ & $\mathrm{~T}$ & $\begin{array}{c}\rightarrow \leftarrow \\
I_{i}^{3}\end{array}$ & $\mathrm{U}$ & $\begin{array}{l}\rightarrow \leftarrow \\
I_{i}^{4}\end{array}$ & $\mathrm{HU}$ & $\overrightarrow{I_{i}^{5}}$ \\
\hline Robust stability & $J_{1}$ & -10 & & -0.01 & & -0.005 & & -0.001 & & -0.0005 & & -0.0001 \\
\hline Maximum control effort & $J_{2}$ & 0.0 & & 0.85 & & 0.9 & & 1.0 & & 1.5 & & 2.0 \\
\hline Worst case settling time & $J_{3}$ & 0.0 & & 14.0 & & 20.0 & & 30.0 & & 35.0 & & 40.0 \\
\hline Noise sensitivity & $J_{4}$ & 0.0 & & 0.5 & & 0.9 & & 1.2 & & 1.4 & & 1.5 \\
\hline Nominal control effort & $J_{5}$ & 0.0 & & 0.5 & & 0.7 & & 1.0 & & 1.5 & & 2.0 \\
\hline Nominalsettling time & $J_{6}$ & 0.0 & & 10.0 & & 11.0 & & 15.0 & & 20.0 & & 25.0 \\
\hline
\end{tabular}

Table 3: Preference range for three-bars ACC benchmark as a MOP.

455

The visualization of the hypercube can be improve using the composed norm. In both Pareto front (Figure 21) and set (Figure 22) the hypercube is easily located (see grayed areas). Now the color could be used for other purpose in order to improve the interpretability. In the example the color is used to make easier point localization, each point of the Pareto set and its corresponding Pareto front values has the same color at all the diagrams. 

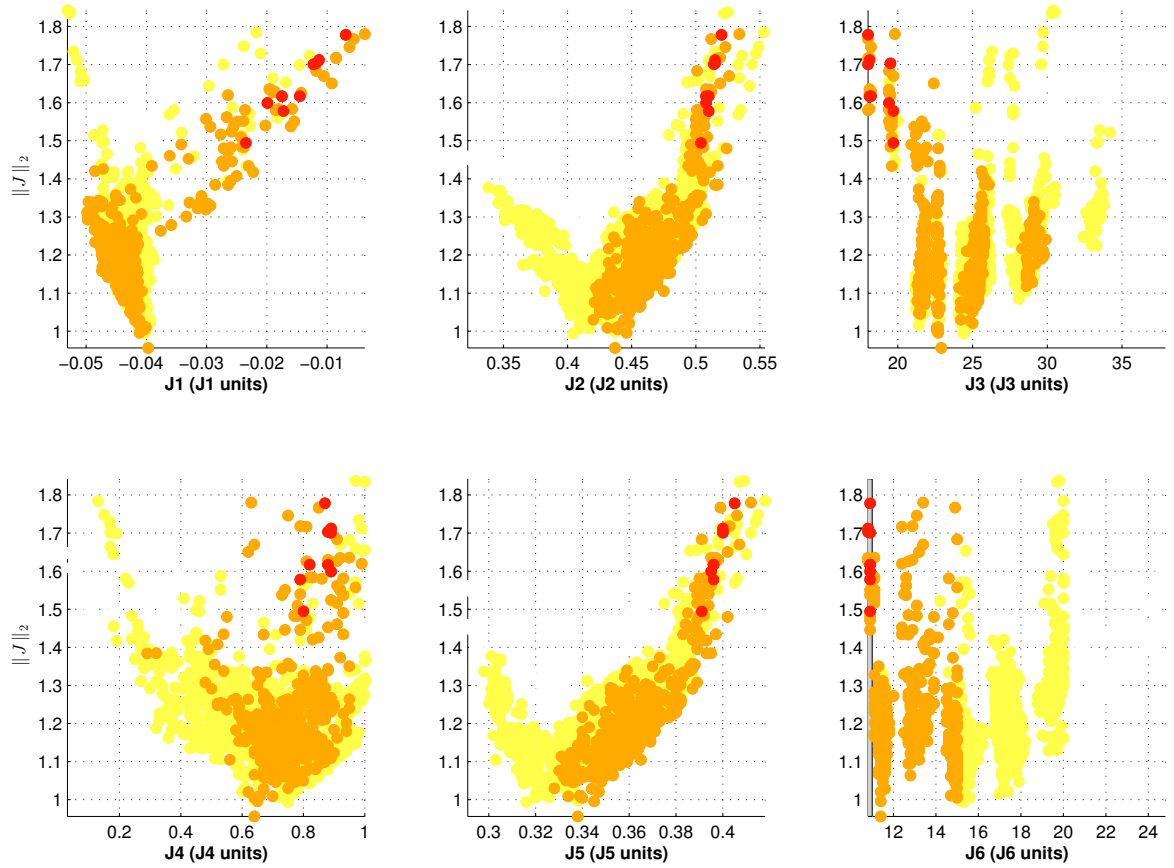

Figure 19: Level diagram representation of the Pareto front for ACC benchmark problem. $\|\hat{J}\|_{2}$ for y-axis synchronization. Colored according to preference ranges using asymmetrics norms. The grayed areas shows the limit of the different preference intervals
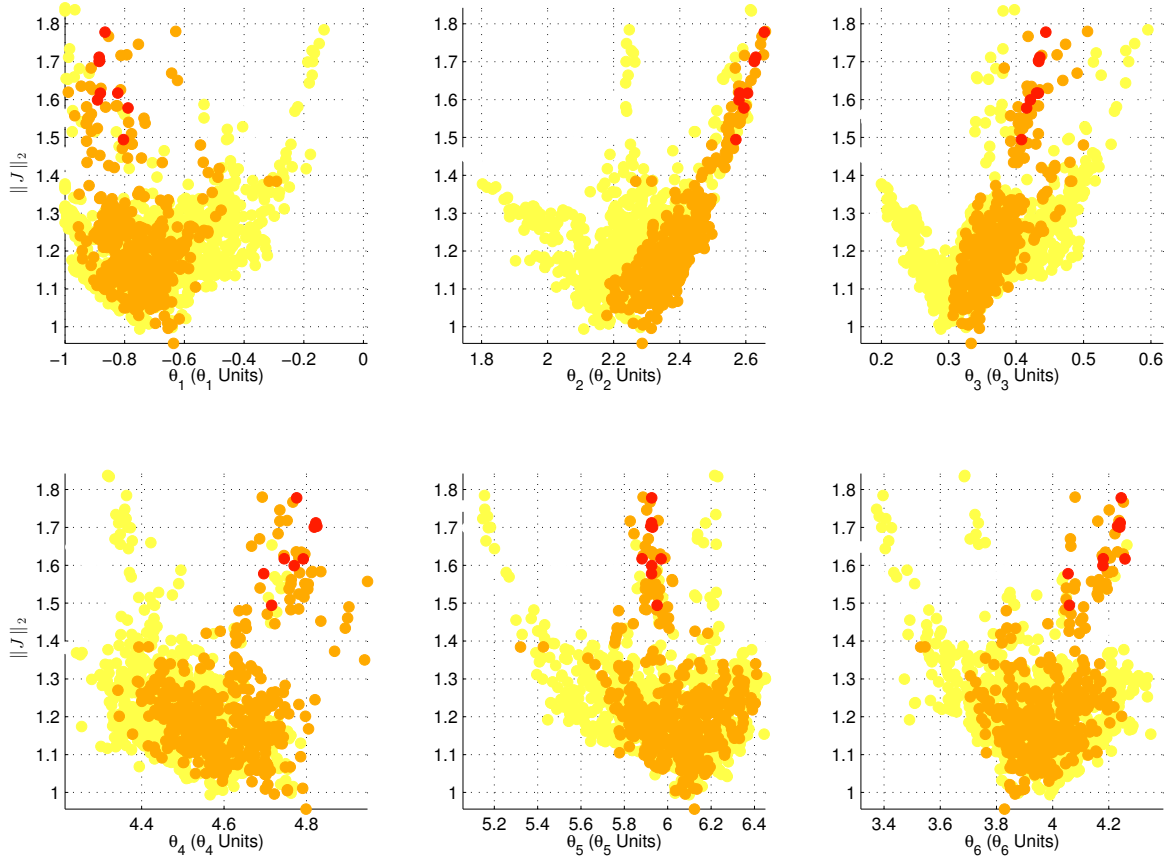

Figure 20: Level diagram representation of the Pareto set for ACC benchmark problem. $\|\hat{J}\|_{2}$ for y-axis synchronization. Colored according to preference ranges using asymmetrics norms. 

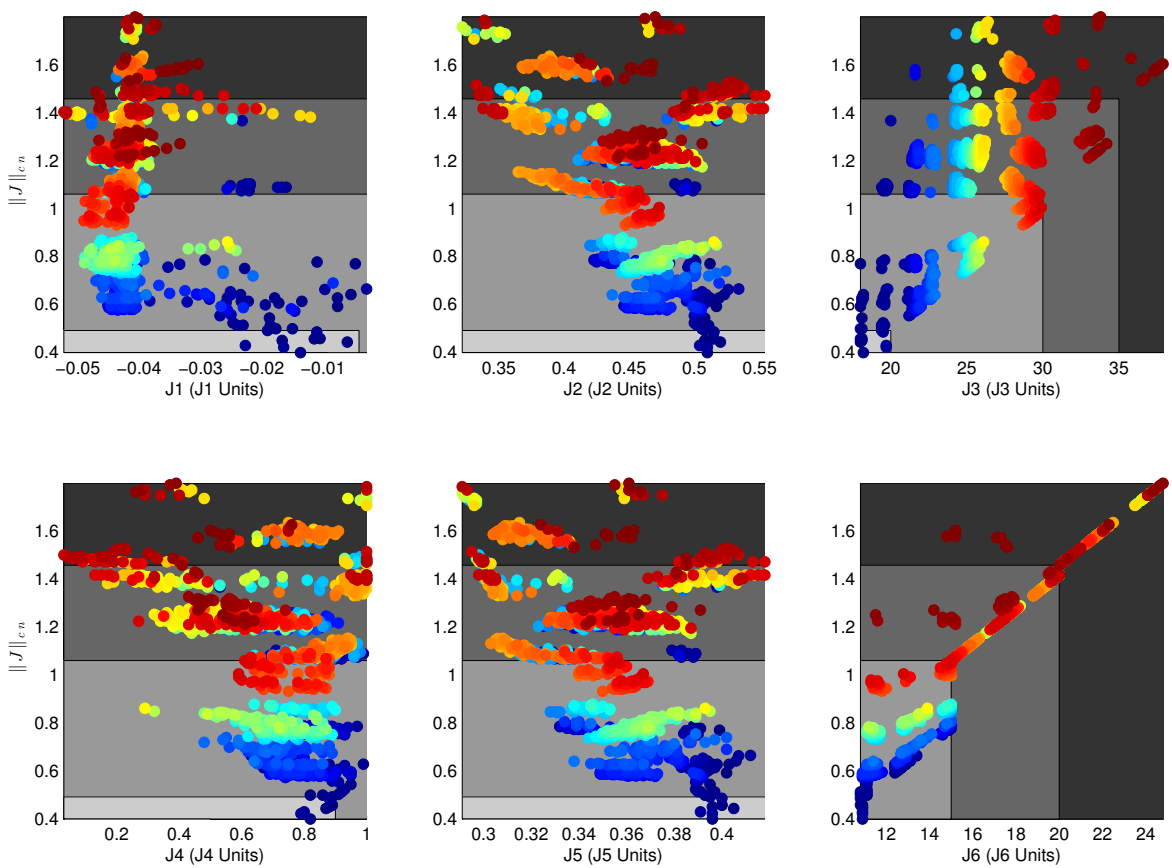

Figure 21: Level diagram representation of the Pareto front for ACC benchmark problem. $\|J\|_{c n}$ for y-axis synchronization. Each Pareto point (front and set) has the same color at each axes.The grayed areas show the different preference intervals (hypercubes).
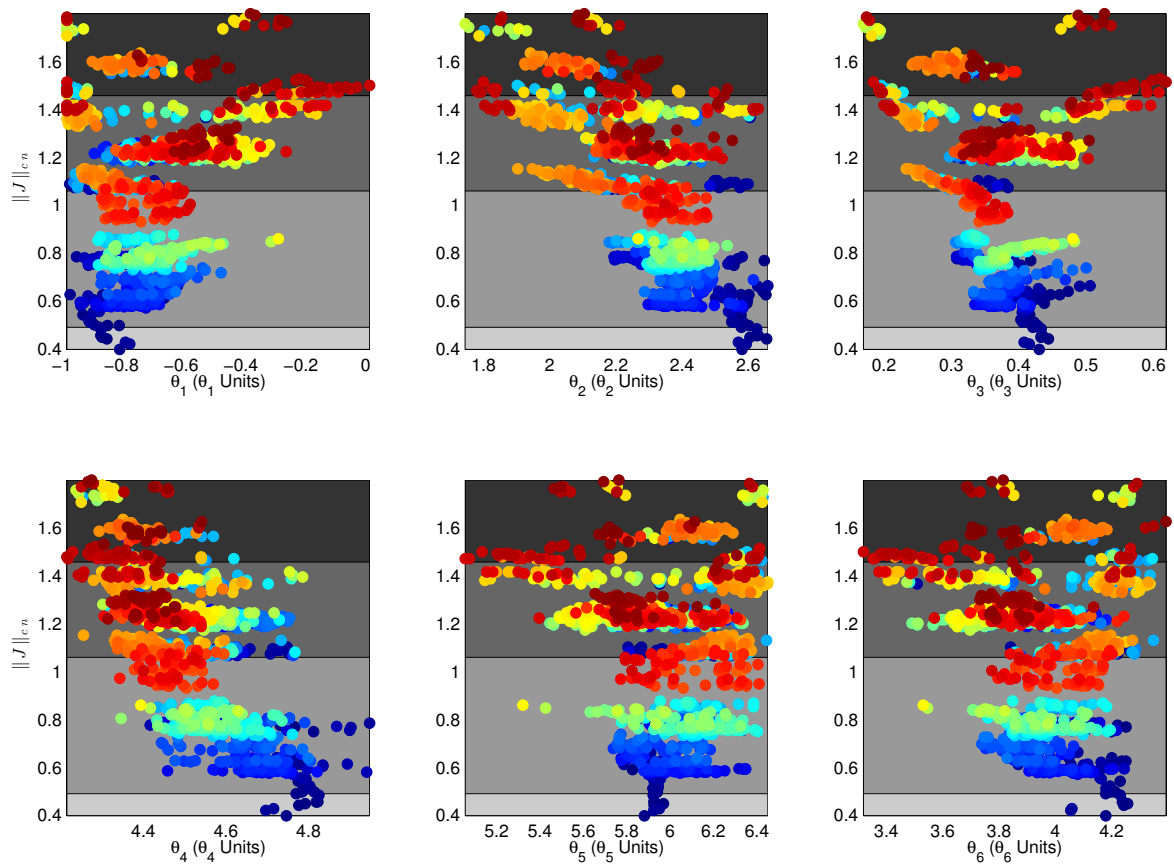

Figure 22: Level diagram representation of the Pareto set for ACC benchmark problem. $\|J\|_{c n}$ for y-axis synchronization. Each Pareto point (front and set) has the same color at each axes. The grayed areas show the different preference intervals (hypercubes). 


\subsection{Pollution monitoring problem}

This last example is a multiobjective problem described in [33] and it is related to locating a pollution monitoring station in a two-dimensional decision space. The five criteria correspond to the expected information loss as estimated by five different experts. The formulation of the problem is based on the following expressions:

$$
\begin{aligned}
f\left(\theta_{1}, \theta_{2}\right) & =-u_{1}\left(\theta_{1}, \theta_{2}\right)-u_{2}\left(\theta_{1}, \theta_{2}\right)-u_{3}\left(\theta_{1}, \theta_{2}\right)+10 \\
u_{1}\left(\theta_{1}, \theta_{2}\right) & =3\left(1-\theta_{1}\right)^{2} e^{-\theta_{1}^{2}-\left(\theta_{2}+1\right)^{2}} \\
u_{2}\left(\theta_{1}, \theta_{2}\right) & =-10\left(\theta_{1} / 4-\theta_{1}^{3}-\theta_{2}^{5}\right) e^{-\theta_{1}^{2}-\theta_{2}^{2}} \\
u_{3}\left(\theta_{1}, \theta_{2}\right) & =1 / 3 \cdot e^{-\left(\theta_{1}+1\right)^{2}-\theta_{2}^{2}}
\end{aligned}
$$

The derived 5 objectives are:

$$
\begin{aligned}
& J_{1}\left(\theta_{1}, \theta_{2}\right)=f\left(\theta_{1}, \theta_{2}\right) \\
& J_{2}\left(\theta_{1}, \theta_{2}\right)=f\left(\theta_{1}-1.2, \theta_{2}-1.5\right) \\
& J_{3}\left(\theta_{1}, \theta_{2}\right)=f\left(\theta_{1}+0.3, \theta_{2}-3.0\right) \\
& J_{4}\left(\theta_{1}, \theta_{2}\right)=f\left(\theta_{1}-1.0, \theta_{2}+0.5\right) \\
& J_{5}\left(\theta_{1}, \theta_{2}\right)=f\left(\theta_{1}-0.5, \theta_{2}-1.7\right)
\end{aligned}
$$

Decision space is constrained to: $\theta_{1} \in[4.9,3.2], \theta_{2} \in[3.5,6]$. An evolutionary multiobjective optimization algorithm is executed obtaining an approximation for the Pareto set (and front) of 790 points.

To illustrate the graphical visualization based on composed norm, the preference table is

\begin{tabular}{|c|c|c|c|c|c|c|c|c|c|c|c|}
\hline & $\overleftarrow{I_{i}^{o}}$ & HD & $\begin{array}{c}\rightarrow \leftarrow \\
I_{i}^{1}\end{array}$ & $\mathrm{D}$ & $\begin{array}{c}\rightarrow \leftarrow \\
I_{i}^{2}\end{array}$ & $\mathrm{~T}$ & $\begin{array}{c}\rightarrow \leftarrow \\
I_{i}^{3} \\
\end{array}$ & $\mathrm{U}$ & $\begin{array}{c}\rightarrow \leftarrow \\
I_{i}^{4}\end{array}$ & $\mathrm{HU}$ & $\overrightarrow{I_{i}^{5}}$ \\
\hline$J_{1}$ & 1 & & 7 & & 9 & & 10 & & 11 & & 12 \\
\hline$J_{2}$ & 1 & & 7 & & 8 & & 9 & & 10 & & 12 \\
\hline$J_{3}$ & 1 & & 7 & & 9 & & 10 & & 11 & & 12 \\
\hline$J_{4}$ & 1 & & 7 & & 8 & & 9 & & 10 & & 12 \\
\hline$J_{5}$ & 1 & & 7 & & 9 & & 10 & & 11 & & 12 \\
\hline
\end{tabular}
shown at Table 4. The composed norm is computed for the approximated Pareto front and used to synchronize Level Diagram axis. The results are shown at Figures 23 and 24. As in the previous example the color is used to make easier point localization, each point of the Pareto set and its corresponding Pareto front values has the same color at all the diagrams.

It can be see that the range of preferences of each point are quickly located, each range correspond to a grayed area. It's easy to see that the best hypercube correspond to Tolerable range. Other type of data analysis can be done, for instance, it is possible to see that very good points for $J 1$ (some of the dark blue points) are not inside Tolerable range. These points

Table 4: Preference range for Pollution MOP problem. 

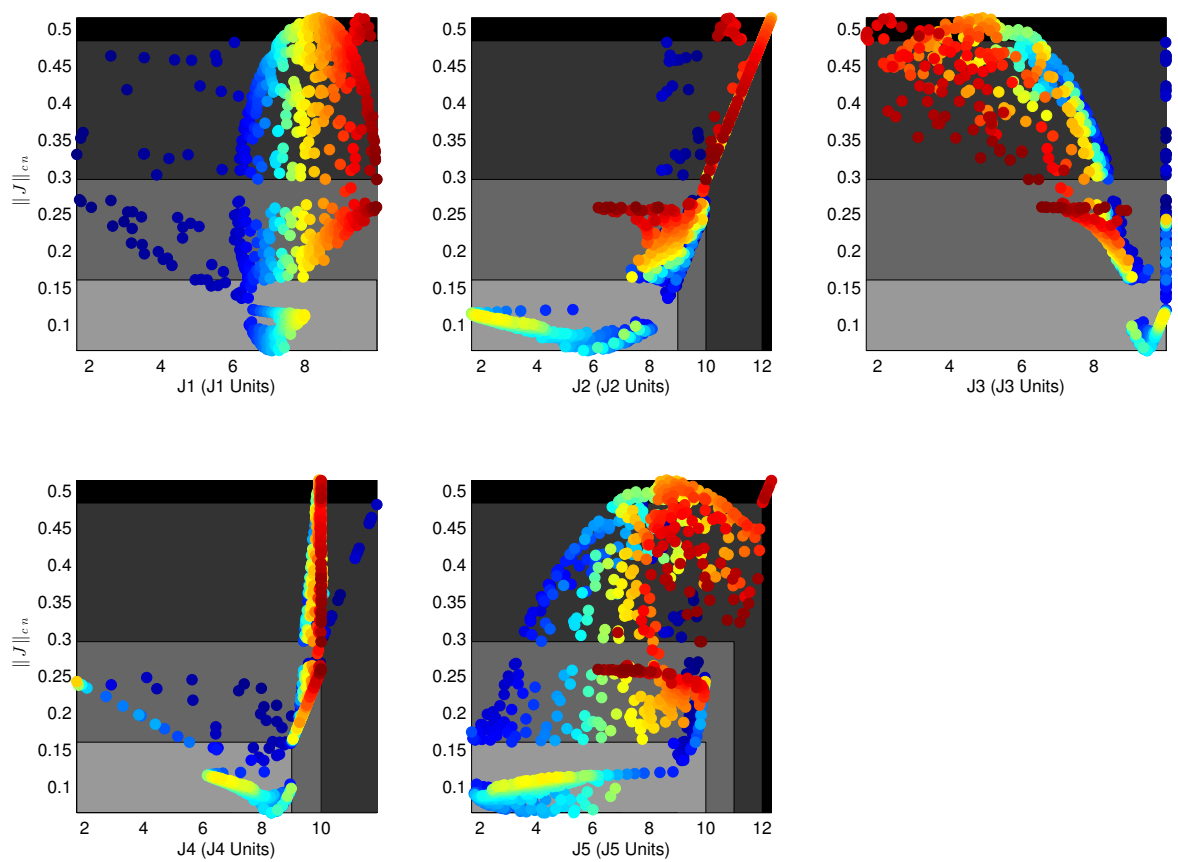

Figure 23: Level diagram representation of the Pareto front for the pollution problem. $\|J\|_{c n}$ for y-axis synchronization. Each Pareto point (front and set) has the same color at each axes. The grayed areas show the different preference intervals (hypercubes).
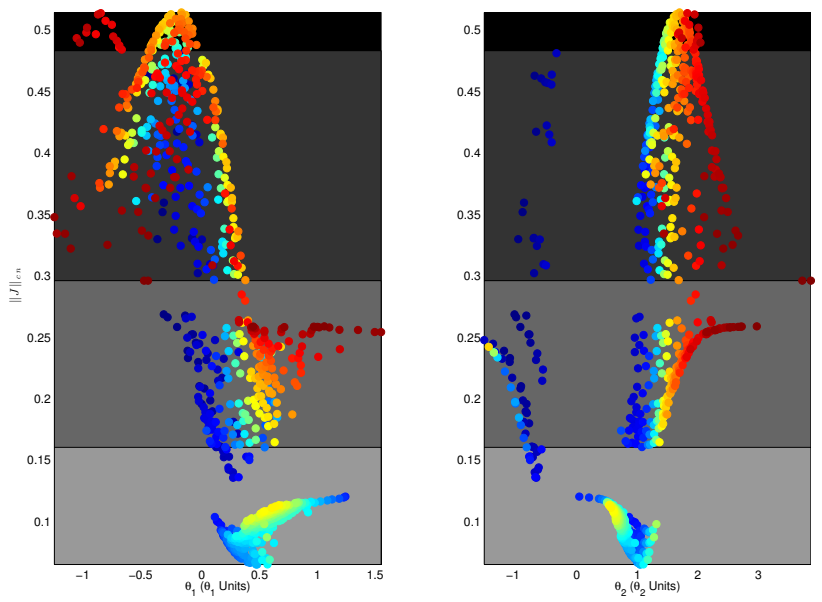

Figure 24: Level diagram representation of the Pareto set for the pollution problem. $\|J\|_{c n}$ for y-axis synchronization. Each Pareto point (front and set) has the same color at each axes. The grayed areas show the different preference intervals (hypercubes). 
are also in contradiction with objective $J 3$, and have parameter values: $-0.5 \leq \theta_{1} \leq 0.5$ and $\theta_{1} \leq 1.4$, etc. Additionally, the hypercube membership is easily translated to the parameter

space. In this example the units of the parameters are a physical position coordinates of a pollution monitoring station, then the designer can easily see that the Tolerable solutions (according to his preferences) are solutions around $\theta_{1} \approx 0.5$ and $\theta_{2} \approx 1$. Inside the Tolerable hypercube, the yellow solutions $\theta_{1} \approx 0.6$ and $\theta_{2} \approx 0.6$ seems a good solution: $J 1 \approx 8$, $J 2 \approx 2.5, J 3 \approx 10, J 4 \approx 7$ and $J 5 \approx 4$.

A detailed analyze of these type of colored Level diagrams shows that several relations between point can easily be extracted. The use of Level diagrams helps the analyze showing clearly the values in the units of the objectives and parameters, giving the designer an engineering point of view. Remark that the use of the composed norm for axis synchronization allows coloring each point for particular purposes (in the example the colors are used to quickly localize a single point in all the diagrams) increasing the amount of available information shown at the diagrams. With more information available the DM can better compare and select the final solution.

\section{Conclusions}

In this work, asymmetric norm is presented as a promising framework to assist graphical visualization Decision Making procedures for high multidimentional MOPs (mainly more than 2 dimensions). It has been shown that asymmetric norm includes dominance characteristics as well as geometrical distance. The computation of asymmetric norm is easy and "cheap" and then it could effectively be included in interactive visualization tools. Several options to use this norm have been presented: coloring points of a Pareto set or defining a composed norm for level diagram synchronization. This last possibility is used when a table of preferences is defined and allows a quick view of the hypervolume membership in large set of Pareto points. It has been demonstrated that the composed norm increase the completeness and persistency of the Level Diagrams, and consequently it helps the DM to better decide between design alternatives and to better understand the multiobjective problem he faces.

Additionally, as asymmetric norm has a complete theory and it is receiving constant improvements in formalization and usability, it seems it could offer more interesting options for multiobjective optimization and decision making.

\section{Acknowledgment}

This work was partially supported by EVO-CONTROL project (ref. PROMETEO/2012/028, Generalitat Valenciana - Spain) and the National Council of Scientific and Technologic Development of Brazil (CNPq) with the postdoctoral fellowship BJT-304804/2014-2.

\section{References}

[1] Alegre, C., Ferrando, I., García-Raffi, L., and Sánchez Pérez, E. (2008). Compactness in asymmetric normed spaces. Topology and its Applications, 155(6):527-539. 
[2] Behzadian, M., Otaghsara, S. K., Yazdani, M., and Ignatius, J. (2012). A state-of the-art survey of TOPSIS applications. Expert Systems with Applications, 39(17):13051 - 13069.

[3] Blasco, X., Herrero, J. M., Sanchis, J., and Martínez, M. (2008). A new graphical visualization of n-dimensional Pareto front for decision-making in multiobjective optimization. Information Sciences, 178(20):3908 - 3924.

[4] Bonissone, P., Subbu, R., and Lizzi, J. (2009). Multicriteria decision making (MCDM): a framework for research and applications. IEEE Computational Intelligence Magazine, $4(3): 48-61$.

[5] Cela, R. and Bollaín, M. (2012). New cluster mapping tools for the graphical assessment of non-dominated solutions in multi-objective optimization. Chemometrics and Intelligent Laboratory Systems, 114(0):72 - 86.

[6] Choi, H. (2014). Data visualization for asymmetric relations. Neurocomputing, 124(0):97 $-104$.

[7] Cobzas, S. (2012). Functional analysis in asymmetric normed spaces. Springer.

[8] Coello Coello, C. A. and Lamont, G. B. (2004). Applications of Multi-Objective evolutionary algorithms. World scientific publishing, advances in natural computation vol. 1 edition.

[9] Coello Coello, C. A., Van Veldhuizen, D., and Lamont, G. (2002). Evolutionary algorithms for solving multi-objective problems. Kluwer Academic press.

[10] Conradie, J. J. and Mabula, M. D. (2013). Completeness, precompactness and compactness in finite-dimensional asymmetrically normed lattices. Topology Appl., 160:2012-2024.

[11] Deb, K., Bandaru, S., Greiner, D., Gaspar-Cunha, A., and Tutum, C. C. (2014). An integrated approach to automated innovization for discovering useful design principles: Case studies from engineering. Applied Soft Computing, 15(0):42 - 56.

[12] Figueira, J., Greco, S., and Ehrgott, M. (2005). Multiple criteria decision analysis: State of the art surveys. Springer international series.

[13] García-Raffi, L., Romaguera, S., and Sánchez-Pérez, E. (2002a). The bicompletion of an asymmetric normed linear space. Acta Mathematica Hungarica, 97(3):183-191.

[14] García-Raffi, L., Romaguera, S., and Sánchez-Pérez, E. (2002b). Sequence spaces and asymmetric norms in the theory of computational complexity. Mathematical and computer modelling, 36(1):1-11.

[15] García-Raffi, L., Romaguera, S., and Sánchez-Pérez, E. (2003). On hausdorff asymmetric normed linear spaces. Houston J. Math, 29(3):717-728. 
[16] Gordo, A., Perronnin, F., Gong, Y., and Lazebnik, S. (2014). Asymmetric distances for binary embeddings. Pattern Analysis and Machine Intelligence, IEEE Transactions on, $36(1): 33-47$.

[17] Inselberg, A. (1985). The plane with parallel coordinates. The Visual Computer, 1:6991.

[18] Inselberg, A. (2009). Parallel Coordinates: Visual Multidimensional Geometry and Its Applications. Springer.

[19] Jonard-Pérez, N. and Sánchez-Pérez, E. A. (2014). Compact convex sets in 2dimensional asymmetric normed lattices. arXiv:1403.6986 [math.GN].

[20] Jonard-Pérez, N. and Sánchez-Pérez, E. A. (2014). Extreme points and geometric aspects of compact convex sets in asymmetric normed spaces. arXiv:1404.0500 [math.FA].

[21] Lotov, A. and Miettinen, K. (2008). Visualizing the Pareto frontier. In Branke, J., Deb, K., Miettinen, K., and Slowinski, R., editors, Multiobjective Optimization, volume 5252 of Lecture Notes in Computer Science, pages 213-243. Springer Berlin / Heidelberg.

[22] Martín-Merino, M. and Muñoz, A. (2005). Visualizing asymmetric proximities with som and mds models. Neurocomputing, 63:171-192.

[23] Martínez, M., Herrero, J., Sanchis, J., Blasco, X., and García-Nieto, S. (2009). Applied pareto multi-objective optimization by stochastic solvers. Engineering Applications of Artificial Intelligence, 22:455 - 465.

[24] Mayor, G. and Valero, O. (2010). Aggregation of asymmetric distances in computer science. Information Sciences, 180(6):803 - 812. Special Issue on Modelling Uncertainty.

[25] Messac, A., Ismail-Yahaya, A., and Mattson, C. (2003). The normalized normal constraint method for generating the pareto frontier. Struct Multidisc Optim, 25:86-98.

[26] Miettinen, K. M. (1998). Nonlinear multiobjective optimization. Kluwer Academic Publishers.

[27] Munro, M. and Aouni, B. (2012). Group decision makers' preferences modelling within the goal programming model: An overview and a typology. Journal of Multi-Criteria Decision Analysis, 19(3-4):169-184.

[28] Olszewski, D. and Šter, B. (2014). Asymmetric clustering using the alpha-beta divergence. Pattern Recognition, 47(5):2031 - 2041.

[29] Reynoso Meza, G. (2014). Controller Tuning by Means of Evolutionary Multiobjective Optimization: a Holistic Multiobjective Optimization Design Procedure. PhD thesis, Universitat Politècnica de València, Spain. 
[30] Reynoso-Meza, G., Blasco, X., Sanchis, J., and Herrero, J. M. (2013). Comparison of design concepts in multi-criteria decision-making using level diagrams. Information Sciences, 221:124 - 141 .

[31] Romaguera, S., Garcia-Raffi, L., Sanchez-Perez, E., and de Caminos, E. (2003). The dual space of an asymmetric normed linear space. Quaestiones Mathematicae, 26(1):82-96.

[32] Sanchis, J., Martínez, M., and Blasco, X. (2008). Multi-objective multiobjective engineering design using preferences. Engineering Optimization, 40(3):253-269.

[33] Thiele, L., Miettinen, K., Korhonen, P., and Molina, J. (2009). A preference-based evolutionary algorithms for multi-objective optimization. Evol. Comput., 3:411 - 436.

[34] Tušar, T. and Filipič, B. (2014). Visualization of pareto front approximations in evolutionary multiobjective optimization: A critical review and the prosection method. IEEE Transactions on Evolutionary Computation, 19.

[35] Wie, B. and Bernstein, D. S. (1992). Benchmark problems for robust control design. Journal of Guidance, Control and Dynamics., 5(15):1057 - 1059.

[36] Zhu, J. and Hipel, K. W. (2012). Multiple stages grey target decision making method with incomplete weight based on multi-granularity linguistic label. Information Sciences, $212: 15-32$.

\section{Appendix A. Asymmetric normed spaces}

Let us explain some definitions and notations regarding topology, metric properties and order properties on linear spaces $\mathbb{R}^{n}$ that are used in this paper. Let $X$ be a linear space. We will write $x$ and $y$ for the elements of the space; note that in the framework of the optimization explained in the previous subsection, these points are in the range of $\boldsymbol{J}$, that is, typically there are $\boldsymbol{\theta}^{1}$ and $\boldsymbol{\theta}^{2}$ of the domain space such that $\boldsymbol{J}\left(\boldsymbol{\theta}^{1}\right)=x$ and $\boldsymbol{J}\left(\boldsymbol{\theta}^{2}\right)=y$. An asymmetric normed space is a real vector space $X$ endowed with a topology that is defined by what is called an asymmetric norm $q$. A function $q: X \rightarrow[0, \infty)$ satisfying

1. $q(t x)=t q(x)$ for every $t \geq 0$ and $x \in X$,

2. $q(x+y) \leq q(x)+q(y)$ and

3. $q(x)=0=q(-x)$ if and only if $x=-x=0$.

is an asymmetric norm. In particular, a standard norm in a Banach space is an asymmetric norm, but the asymmetric lattice norm associated to the usual order in $\mathbb{R}^{n}$ that will be used in the present paper is not a norm (see below).

An asymmetric norm induces a non symmetric topology on $X$ that is generated by the quasi-metric $d(x, y)=q(y-x), x, y \in X$. That is, the asymmetric open balls $B_{q}(x, \varepsilon)=$ $\{y \in X \mid q(y-x)<\varepsilon\}$ define a topology on $X$, and so the asymmetric normed space $(X, q)$ can be considered as a topological space. This topology satisfies the separation axiom $T_{0}$, 
and the vector sum of $X$ is continuous with respect to, but in general this topology is not Hausdorff and the multiplication by scalars on $X$ is not continuous. Thus $(X, q)$ fails to be a topological vector space in the classical sense.

Any asymmetric normed space $(X, q)$ has a (symmetric standard) norm associated. That is the norm $q^{s}: X \rightarrow[0, \infty)$ defined by the formula

$$
q^{s}(x):=\max \{q(x), q(-x)\}, \quad x \in X .
$$

In this paper we have used a particular construction for defining asymmetric norms based in the lattice structure of $\mathbb{R}^{n}$. Recall that a vector lattice $X$ is a linear space with an order $\leq$ for which the supremum $x \vee y$ and the infimum $x \wedge y$ are defined as elements of $x$ for each couple $x, y \in X$, and so also the modulus $|x|$ of each element $x \in X$. A Banach lattice is a vector lattice $(X, \leq)$ that is also a Banach space $(X,\|\cdot\|)$ such that the norm is compatible with the order $\leq$, that is, $\|x\| \leq\|y\|$ whenever $|x| \leq|y|$. Consider the coordinatewise order $\leq$ on $\mathbb{R}^{n}$ and fix a norm $\|\cdot\|$ on $\mathbb{R}^{n}$ - for example the Euclidean norm, but not necessarilythat is compatible with $\leq$. We write $\left(\mathbb{R}^{n}\right)^{+}$for the positive cone, that is, all the elements that are coordinatewise bigger or equal than 0 . We consider the asymmetric norm that is canonically defined by using the Banach lattice structure of $\mathbb{R}^{n}$ as

$$
q(x):=\|x \vee 0\|, \quad x \in \mathbb{R}^{n} .
$$

For the particular case of $\|\cdot\|$ being Euclidean norm, we obtain the definition used in the paper. General results on asymmetric normed spaces can be found in [7, 14, 13, 15, 31. Specific results on fundamental topological properties that are necessary for obtaining approximation tools are nowadays also known, also for the case of lattice asymmetric norms as the ones that we have defined (see [1, 10, 19, 20]). 